\title{
PROPAGATION OF CRACKS IN ELASTIC MEDIA
}

\author{
By \\ Avner Friedman \\ and \\ Yong Liu
}

IMA Preprint Series \# 1290

February 1995

INSTITUTE FOR MATHEMATICS AND ITS APPLICATIONS UNIVERSITY OF MINNESOTA

514 Vincent Hall

206 Church Street S.E.

Minneapolis, Minnesota 55455 


\title{
Propagation of Cracks in Elastic media
}

\author{
Avner Friedman* and Yong Liu ${ }^{\dagger}$
}

\section{Introduction}

In this paper we study a quasi-stationary model of crack propagation in $2 d$ elastic medium. The model is introduced in $\S 1$. The stress function $\phi$ satisfies the biharmonic equation away from the crack $\Gamma(t)$, the free boundary conditions are

$$
\phi=\frac{\partial \phi}{\partial n}=0
$$

from both sides of $\Gamma(t)$, and

$$
\dot{X}(t)=\Phi(|\dot{X}(t)|) \vec{J}(X(t))
$$

where $X(t)$ is the moving tip of the crack, i.e., the moving end-point of $\Gamma(t)$; the other end-point of $\Gamma(t)$ is fixed at the boundary. Here $\Phi(s)$ is a rather simple explicit function, and $\vec{J}(X)$ is the so-called $J$-integral. The $J$-integral depends on the stress tensor in a global way.

The dynamic condition (0.1) expresses the physical fact that as the tip of the crack propagates, energy flows from the stress field in the body to the crack tip.

In $\S 2$ we compute the $J$-integral explicitly in terms of $\phi$. In $\S 3$ we establish some results on biharmonic functions in a domain $D \backslash \Gamma_{0}$ where $D$ is the strip $\{(x, y) ; x \in \mathbb{R},-a<y<a\}$ and $\Gamma_{0}=\{(x, 0) ;-\infty<x<0\}$; we study the Dirichlet problem as well as the singularity at the tip $(0,0)$. These results are needed in the following sections.

In $\S 4$ we construct a travelling wave solution to the crack propagation problem in $D$. The solution is symmetric in $y$, and the crack propagates along the $x$-axis, from $x=-\infty$, at a uniform speed. The rest of the paper is concerned with a small perturbation about the travelling wave solution. In $\S 5$ we introduce the linearization of the nonlinear perturbed problem. This linearized problem is studied in sections 6-9. In $\S 8$ we prove global existence and uniqueness. In sections 8 and 9 we show that if $(x(t), y(t))$ is the tip of the crack for the linearized problem, then $\dot{y}(0+)>0$ (we also compute $\dot{y}(0+)$ explicitly). The proofs require some auxiliary estimates and these are proved in sections 6,7 .

\footnotetext{
${ }^{*}$ Institute for Mathematics and its Applications, University of Minnesota, Minneapolis, Minnesota 55455. Supported partially by National Science Foundation Grant DMS 87-22187.

${ }^{\dagger}$ School of Mathematics, University of Minnesota, Minneapolis, Minnesota 55455.
} 


\section{The crack model}

Let $\Omega$ be a domain in $\mathbb{R}^{2}$, representing a homogeneous elastic body. Let $u=\left(u_{i}\right)$, $\epsilon=\left(\epsilon_{i j}\right)$ and $\sigma=\left(\sigma_{i j}\right)$ denote the displacement vector, the strain tensor and the stress tensor, respectively. The linear elasticity equations for homogeneous isotropic material consist of the constitutive law

$$
\sigma_{i j}=\frac{E}{1+\nu}\left(\epsilon_{i j}+\frac{\nu}{1-2 \nu} \epsilon_{k k} \delta_{i j}\right)
$$

and the equilibrium conditions

$$
\frac{\partial}{\partial x_{j}} \sigma_{i j}=0
$$

provided no body forces are assumed. Here $E$ is the Young modulus, and $\nu$ is the Poisson ratio, and the strain-displacement relation is given by

$$
\epsilon_{i j}=\frac{1}{2}\left(u_{i, j}+u_{j, i}\right), \quad u_{i, j}=\partial_{j} u_{i}
$$

Suppose there is initially a crack in $\Omega$, given by a non-intersecting curve $\Gamma_{0}$ with initial point on $\partial \Omega$ and terminal point (the "crack tip") $X_{0}=\left(x_{0}, y_{0}\right)$ inside $\Omega$. Under external forces the crack tip will generally propagate, and we shall denote it by $X(t)$. The crack propagation problem consists of finding the displacement $u$ and path $X(t)$ such that

$$
\sigma_{i j, j}=0 \text { in } \Omega \backslash \Gamma(t)
$$

where

$$
\begin{gathered}
\Gamma(t)=\Gamma_{0} \cup\{X=X(s), 0 \leq s \leq t\}, \\
\sigma_{i j} n_{j}=0 \text { on } \Gamma^{ \pm}(t) \text { (no traction on } \Gamma^{ \pm}(t) \text { ) }
\end{gathered}
$$

where $\Gamma^{ \pm}(t)$ means both sides of $\Gamma(t)$, and $\left(n_{j}\right)$ is the normal to the curve,

$$
u_{i}=\beta_{i} \text { on } \partial_{1} \Omega, \sigma_{i j} n_{j}=g_{i} \text { on } \partial_{2} \Omega
$$

where $\partial \Omega$ is a disjoint union of $\partial_{1} \Omega, \partial_{2} \Omega$, and

$$
\text { an appropriate dynamical equation holds for } X(t), X(0)=X_{0} \text {. }
$$

The dependence on $t$ appears only through the dynamical equation for $X(t)$; the elasticity equations are taken to be time-independent. Thus the model we consider is "quasistationary."

To obtain explicit formula for (1.8) we begin with the energy criterion given by Griffith [7]. Accordingly, when a crack is extended, there is a flow of energy from the stress field in the body to the crack tip. This energy is then stored on both faces of the newly enlarged crack, and

$$
G=\gamma
$$

where $G$ is the energy release rate at the crack tip and $\frac{1}{2} \gamma$ is the stored fracture energy (which in the $3 d$ case is also called the surface energy). 
There is no satisfactory theory for determining $\gamma$ from basic principles. It is believed that crack propagation proceeds in two stages. In the first stage the speed $v$ of the crack tip increases until it reaches a limit $v_{\infty}$, and in the second stage the crack tip continues to move with the uniform speed $v_{\infty}$; we refer to Freund [4] for very detailed explanations and for references. We also refer to a recent work by Slepyan [12] based on a variational principle which explains what happens at the limit speed.

Here we shall deal only with the first stage (i.e., $v<v_{\infty}$ ). For some materials [4, p.417],

$$
\gamma=\gamma_{0}\left(\frac{v_{\infty}+v}{v_{\infty}-v}\right)^{m}
$$

where $m$ is a positive integer, and $m, \gamma_{0}, v_{\infty}$ depend on the material. For simplicity we shall henceforth take $m=1$, i.e.,

$$
\gamma=\gamma_{0} \frac{v_{\infty}+v}{v_{\infty}-v}
$$

all our results, however, extend to the case of any $m>0$.

We next turn to the energy release rate $G$. Following Rice [11], $G$ can be described in terms of the $J$-integral, which we proceed to define. Let $P$ be any point in $\Omega$ and let $\Lambda$ be any smooth non-intersecting curve in $\Omega$ surrounding $P$. The $J$-integral is defined by

$$
\overrightarrow{J_{\Lambda}}(P)=\int_{\Lambda}(W \vec{n}-\vec{s} \cdot D u)
$$

where $\vec{n}$ is the outward normal, $W$ is the strain energy density given by

$$
W=\frac{1}{2} \sigma_{i j} \epsilon_{i j}
$$

and

$$
\vec{s}=\left(s_{i}\right)=\left(\sigma_{i j} n_{j}\right)
$$

is the traction vector.

By the divergence theorem one can easily verify (cf. [8]) that

$$
\int_{\gamma}(W \vec{n}-\vec{s} \cdot D u)=0
$$

if $\gamma$ is any curve in $\Omega$ such that the region enclosed by $\gamma$ does not contain cracks.

Consider now the case where $P$ is the crack tip $X(t)$, and $\Lambda$ is a closed smooth nonintersecting curve with initial and terminal point on the crack $\Gamma(t)$ such that it does not intersect $\Gamma(t)$, except at its endpoints. Denote the diameter of $\Lambda$ by $|\Lambda|$. We take any family of such curves $\Lambda$ with diameter which converges to zero, and let

$$
\vec{J}(P)=\lim _{|\Lambda| \rightarrow 0} \vec{J}_{\Lambda}(P) .
$$

Under some smoothness assumptions on $u$ and $X(t)$ it was shown by Gurtin [8] and Ohtsuka [10] that the limit in (1.14) exists and (motivated by the work of Rice [11]) that the energy release rate $G$ at the crack tip $P$ can be expressed by the $J$-integral as

$$
G=\vec{e} \cdot \vec{J}(P)
$$


where $\vec{e}$ is the direction of motion of the crack tip, i.e.,

$$
G=\frac{\dot{X}(t)}{|\dot{X}(t)|} \cdot \vec{J}(X(t))
$$

Combining this with (1.9) we have

$$
\frac{\dot{X}(t)}{|\dot{X}(t)|} \cdot \vec{J}(X(t))=\gamma .
$$

A necessary condition for (1.16) to hold is that

$$
|\vec{J}(X(t))| \geq \gamma
$$

if $|\vec{J}(X(t))|<\gamma$, the crack does not propagate.

If

$$
|\vec{J}(X(t))|=\gamma
$$

then (1.16) implies that

$$
\dot{X}(t)=\frac{\vec{J}(X(t))}{\gamma}|\dot{X}(t)| .
$$

Stump and Le [13] have recently developed a variational principle and used it to establish that

$$
|\vec{J}(X(t))| \leq \gamma
$$

always holds; furthermore,

if $|\vec{J}(X(t))|<\gamma$ then there is no propagation, whereas

if $|\vec{J}(X(t))|=\gamma$ then the crack tip propagates in the direction $\frac{1}{\gamma} \vec{J}(X(t))$.

Combining this with (1.10) we conclude that

(i) If $|\vec{J}(X(t))| \leq \gamma_{0}$ then the crack does not propagate;

(ii) If $|\vec{J}(X(t))|>\gamma_{0}$ then the crack propagates with speed $v(t)$ and direction $\dot{X}(t) /|\dot{X}(t)|$ determined by

$$
v(t)=v_{\infty} \frac{\left(|\vec{J}(X(t))|-\gamma_{0}\right)_{+}}{|\vec{J}(X(t))|+\gamma_{0}}, \quad v(t)=|\dot{X}(t)|
$$

and

$$
\dot{X}(t)=\frac{v(t)}{\gamma_{0}} \frac{v_{\infty}-v(t)}{v_{\infty}+v(t)} \vec{J}(X(t)) .
$$

Equation (1.22) is the dynamical equation sought in (1.8), but we still need to compute $\vec{J}(X(t))$ more explicitly. This will be done in the next section. 


\section{Computation of $\vec{J}$}

In plane elasticity, $\left(\sigma_{i j}\right)$ satisfies

$$
\frac{\partial \sigma_{11}}{\partial x}+\frac{\partial \sigma_{12}}{\partial y}=0
$$

and

$$
\frac{\partial \sigma_{21}}{\partial x}+\frac{\partial \sigma_{22}}{\partial y}=0
$$

Equations (2.1) and (2.2) imply that there exist functions $\psi_{1}$ and $\psi_{2}$ such that

$$
\begin{aligned}
& \sigma_{11}=\frac{\partial \psi_{1}}{\partial y}, \quad \sigma_{12}=-\frac{\partial \psi_{1}}{\partial x} \\
& \sigma_{21}=\frac{\partial \psi_{2}}{\partial y}, \quad \sigma_{22}=-\frac{\partial \psi_{2}}{\partial x}
\end{aligned}
$$

Since $\sigma_{12}=\sigma_{21}$,

$$
\frac{\partial \psi_{1}}{\partial x}+\frac{\partial \psi_{2}}{\partial y}=0
$$

so that there is a function $\phi$ such that

$$
\psi_{1}=\frac{\partial \phi}{\partial y}, \quad \psi_{2}=-\frac{\partial \phi}{\partial x}
$$

$\phi$ is called the stress function. We can express the $\sigma_{i j}$ in the form

$$
\sigma_{11}=\frac{\partial^{2} \phi}{\partial y^{2}}, \quad \sigma_{12}=-\frac{\partial^{2} \phi}{\partial x \partial y}, \quad \sigma_{22}=\frac{\partial^{2} \phi}{\partial x^{2}} .
$$

The stress tensor $\sigma=\left(\sigma_{i j}\right)$ is uniquely determined by $\phi$ up to an arbitrary linear function.

We rewrite (1.1) in component form:

$$
\begin{aligned}
\sigma_{11} & =\frac{E}{(1+\nu)(1-2 \nu)}\left[(1-\nu) \epsilon_{11}+\nu\left(\epsilon_{22}+\epsilon_{33}\right)\right] \\
\sigma_{22} & =\frac{E}{(1+\nu)(1-2 \nu)}\left[(1-\nu) \epsilon_{22}+\nu\left(\epsilon_{11}+\epsilon_{33}\right)\right] \\
\sigma_{33} & =\frac{E}{(1+\nu)(1-2 \nu)}\left[(1-\nu) \epsilon_{33}+\nu\left(\epsilon_{11}+\epsilon_{22}\right)\right], \\
\sigma_{12} & =\frac{E}{1+\nu} \epsilon_{12}, \quad \sigma_{13}=\frac{E}{1+\nu} \epsilon_{13}, \quad \sigma_{23}=\frac{E}{1+\nu} \epsilon_{23} .
\end{aligned}
$$

Conversely, the strain tensor is given in terms of the stress tensor by

$$
\begin{aligned}
& \epsilon_{11}=\frac{1}{E}\left[\sigma_{11}-\nu\left(\sigma_{22}+\sigma_{33}\right)\right], \\
& \epsilon_{22}=\frac{1}{E}\left[\sigma_{22}-\nu\left(\sigma_{11}+\sigma_{33}\right)\right], \\
& \epsilon_{33}=\frac{1}{E}\left[\sigma_{33}-\nu\left(\sigma_{11}+\sigma_{22}\right)\right],
\end{aligned}
$$




$$
\epsilon_{12}=\frac{1+\nu}{E} \sigma_{12}, \epsilon_{13}=\frac{1+\nu}{E} \sigma_{13}, \epsilon_{23}=\frac{1+\nu}{E} \sigma_{23} .
$$

Substituting (1.3) into the equilibrium equation (1.2) and applying the divergence we find that

$$
\triangle \operatorname{div} u=0 .
$$

In the case of a plane deformation, the last component $u_{3}$ of the displacement $u$ is zero, while the components $u_{1}$ and $u_{2}$ are functions of $x$ and $y$ only, and so $\epsilon_{i 3}=0, \sigma_{13}=\sigma_{23}=0$.

By (2.7) and (2.8),

$$
\begin{aligned}
\triangle \phi=\sigma_{11}+\sigma_{22} & =\frac{E}{(1+\nu)(1-2 \nu)}\left(\epsilon_{11}+\epsilon_{22}\right) \\
& =\frac{E}{(1+\nu)(1-2 \nu)} \operatorname{divu}
\end{aligned}
$$

and therefore (2.10) becomes

$$
\triangle^{2} \phi=0 \text { in } \Omega \backslash \Gamma(t) .
$$

Next we derive boundary conditions for $\phi$. We begin with the free traction condition

$$
\sigma_{i j}^{+} n_{j}=\sigma_{i j}^{-} n_{j}=0 \text { on } \Gamma(t) .
$$

By (2.7),

$$
\begin{aligned}
\sigma_{1 j} n_{j} & =\frac{\partial^{2} \phi}{\partial y^{2}} n_{1}-\frac{\partial^{2} \phi}{\partial x \partial y} n_{2} \\
& =-\frac{\partial}{\partial x}\left(\frac{\partial \phi}{\partial y}\right) \tau_{1}-\frac{\partial}{\partial y}\left(\frac{\partial \phi}{\partial y}\right) \tau_{2}=-\frac{\partial}{\partial \tau}\left(\frac{\partial \phi}{\partial y}\right)
\end{aligned}
$$

where $\vec{\tau}=\left(n_{2},-n_{1}\right)$ is the tangent vector to $\Gamma(t)$.

Similarly,

$$
\sigma_{2 j} n_{j}=-\frac{\partial^{2} \phi}{\partial x \partial y} n_{1}+\frac{\partial^{2} \phi}{\partial x^{2}} n_{2}=\frac{\partial}{\partial \tau}\left(\frac{\partial \phi}{\partial x}\right) .
$$

If $\phi \in C^{1}(\bar{\Omega})$, then $(2.12)$ is equivalent to

$$
\nabla \phi \equiv \text { const. along } \Gamma(t) .
$$

Since the stress $\sigma$ only depends on the second derivatives of $\phi$, any linear addition to $\phi$ will not change $\sigma$.

Let us prescribe a surface force $\vec{s}=\left(s_{1}, s_{2}\right)$ on $\partial \Omega$. Then by the same calculations as in (2.13), (2.14),

$$
s_{1}=\sigma_{1 j} n_{j}=-\frac{\partial}{\partial \tau}\left(\frac{\partial \phi}{\partial y}\right), s_{2}=\sigma_{2 j} n_{j}=\frac{\partial}{\partial \tau}\left(\frac{\partial \phi}{\partial x}\right) .
$$

Hence $\phi_{x}$ and $\phi_{y}$ are determined up to a constant.

We assumed that the crack initiated at the boundary $\partial \Omega$. Hence once we fix the constants for $\phi$ and $\partial \phi / \partial n$ on $\Gamma(t)$, this uniquely determines $\phi$ and $\partial \phi / \partial n$ on $\partial \Omega$. In view of (2.15) we can take

$$
\phi=\frac{\partial \phi}{\partial n}=0 \text { on } \Gamma(t)
$$


Then the boundary conditions on $\partial \Omega$ becomes

$$
\phi=f, \frac{\partial \phi}{\partial n}=g \text { on } \partial \Omega
$$

where $f, g$ are prescribed functions.

Next we shall compute the $J$-integral defined by (1.11) in terms of the stress function $\phi$.

By (2.8) and (2.9),

$$
\sigma_{33}=\frac{\nu E}{(1+\nu)(1-2 \nu)}\left(\epsilon_{11}+\epsilon_{22}\right)=\nu\left(\sigma_{11}+\sigma_{22}\right)=\nu \triangle \phi .
$$

It follows by (2.9), (2.7) and (2.18) that

$$
\begin{aligned}
\epsilon_{11} & =\frac{1}{E}\left[\phi_{22}-\nu\left(\phi_{11}+\nu \Delta \phi\right)\right] \\
& =\frac{1+\nu}{E}\left[(1-\nu) \phi_{22}-\nu \phi_{11}\right] \quad\left(\phi_{11}=\frac{\partial^{2} \phi}{\partial x^{2}}, \phi_{22}=\frac{\partial^{2} \phi}{\partial y^{2}}\right) \\
\epsilon_{22} & =\frac{1}{E}\left[\phi_{11}-\nu\left(\phi_{22}+\nu \Delta \phi\right)\right] \\
& =\frac{1+\nu}{E}\left[(1-\nu) \phi_{11}-\nu \phi_{22}\right] \\
\epsilon_{12} & =-\frac{1+\nu}{E} \phi_{12} \quad\left(\phi_{12}=\frac{\partial^{2} \phi}{\partial x \partial y}\right) .
\end{aligned}
$$

By (1.1) and (1.12), the strain energy density is

$$
\begin{aligned}
W & =\frac{E}{2(1+\nu)}\left(\epsilon_{i j} \epsilon_{i j}+\frac{\nu}{1-2 \nu} \epsilon_{i i} \epsilon_{j j}\right) \\
& =\frac{E}{2(1+\nu)}\left[\epsilon_{11}^{2}+\epsilon_{22}^{2}+2 \epsilon_{12}^{2}+\frac{\nu}{1-2 \nu}\left(\epsilon_{11}+\epsilon_{22}\right)^{2}\right]
\end{aligned}
$$

Using (2.19)-(2.21) we then have

$$
W=\frac{1+\nu}{2 E}\left\{\left[(1-\nu) \phi_{22}-\nu \phi_{11}\right]^{2}+\left[(1-\nu) \phi_{11}-\nu \phi_{22}\right]^{2}+2 \phi_{12}^{2}+\frac{\nu}{1-2 \nu}[(1-\nu) \Delta \phi-\nu \Delta \phi]^{2}\right\}
$$

from which we easily compute that

$$
W=\frac{1+\nu}{2 E}\left\{(1-\nu)(\Delta \phi)^{2}-2 \phi_{11} \phi_{22}+2 \phi_{12}^{2}\right\} .
$$

Next we evaluate $\vec{s} \cdot D u$. We begin by expressing the displacement vector $u$ in terms of $\phi$.

By (2.19) and (2.20),

$$
\frac{\partial u_{1}}{\partial x}=\epsilon_{11}=\frac{1+\nu}{E}\left[-\phi_{11}+(1-\nu) \triangle \phi\right]
$$


and

$$
\frac{\partial u_{2}}{\partial y}=\epsilon_{22}=\frac{1+\nu}{E}\left[-\phi_{22}+(1-\nu) \triangle \phi\right] .
$$

Denote by $(\Delta \phi)^{c}$ the harmonic conjugate of $\Delta \phi$, and define the holomorphic function

$$
\begin{aligned}
\Psi(z) & =\int\left(\Delta \phi+i(\Delta \phi)^{c}\right)(z) d z \\
& =p(z)+i q(z) .
\end{aligned}
$$

Then $p$ and $q$ are harmonic functions, and

$$
\begin{gathered}
\frac{\partial p}{\partial x}=\frac{\partial q}{\partial y}=\Delta \phi \\
\frac{\partial p}{\partial y}=-\frac{\partial q}{\partial x}=-(\triangle \phi)^{c} .
\end{gathered}
$$

Substituting (2.26) into (2.23) and into (2.24), we get

$$
\frac{\partial u_{1}}{\partial x}=\frac{1+\nu}{E}\left(-\phi_{11}+(1-\nu) \frac{\partial p}{\partial x}\right)
$$

and

$$
\frac{\partial u_{2}}{\partial y}=\frac{1+\nu}{E}\left(-\phi_{22}+(1-\nu) \frac{\partial q}{\partial y}\right)
$$

By integration we then have

$$
u_{1}=\frac{1+\nu}{E}\left(-\phi_{1}+(1-\nu) p\right)+h(y)
$$

and

$$
u_{2}=\frac{1+\nu}{E}\left(-\phi_{2}+(1-\nu) q\right)+k(x)
$$

where $h$ and $k$ are arbitrary functions.

By (2.21) and the last two equations,

$$
\begin{aligned}
-\frac{1+\nu}{E} \phi_{12}=\epsilon_{12} & =\frac{1}{2}\left(\frac{\partial u_{1}}{\partial y}+\frac{\partial u_{2}}{\partial x}\right) \\
& =-\frac{1+\nu}{E} \phi_{12}+\frac{1}{2}(1-\nu)\left(\frac{\partial p}{\partial y}+\frac{\partial q}{\partial x}\right)+h^{\prime}(y)+k^{\prime}(x) .
\end{aligned}
$$

Recalling (2.27) we find that

$$
h^{\prime}(y)+k^{\prime}(x)=0 .
$$

Consequently $h^{\prime}(y)=-k^{\prime}(x)=$ const. and thus

$$
h(y)=a y+b, \quad k(x)=-a x+c .
$$

The form of $h$ and $k$ indicate that they represent a rigid displacement and can thus be disregarded in the analysis of deformation. We shall therefore take $h \equiv k \equiv 0$ (without affecting the physical problem). Then (2.30) and (2.31) become

$$
u_{1}=\frac{1+\nu}{E}\left(-\phi_{1}+(1-\nu) p\right),
$$




$$
u_{2}=\frac{1+\nu}{E}\left(-\phi_{2}+(1-\nu) q\right)
$$

We now compute

$$
\begin{aligned}
\vec{s} \cdot D_{1} u & =s_{1} D_{1} u_{1}+s_{2} D_{1} u_{2} \\
& =\frac{1+\nu}{E}\left\{s_{1}\left[-\phi_{11}+(1-\nu) \triangle \phi\right]+s_{2}\left[-\phi_{12}+(1-\nu)(\triangle \phi)^{c}\right]\right\} .
\end{aligned}
$$

Recalling that

$$
\begin{aligned}
& s_{1}=\sigma_{1 j} n_{j}=\phi_{22} n_{1}-\phi_{12} n_{2}, \\
& s_{2}=\sigma_{2 j} n_{j}=-\phi_{12} n_{1}+\phi_{11} n_{2},
\end{aligned}
$$

we have

$$
\begin{aligned}
& \vec{s} \cdot D_{1} u= \frac{1+\nu}{E}\left\{\left(\phi_{22} n_{1}-\phi_{12} n_{2}\right)\left(-\phi_{11}+(1-\nu) \Delta \phi\right)+\right. \\
&\left.\left(-\phi_{12} n_{1}+\phi_{11} n_{2}\right)\left(-\phi_{12}+(1-\nu)(\Delta \phi)^{c}\right)\right\} \\
&= \frac{1+\nu}{E}\left\{-\phi_{11} \phi_{22} n_{1}+\phi_{11} \phi_{12} n_{2}+(1-\nu) s_{1} \Delta \phi+\right. \\
&\left.\phi_{12}^{2} n_{1}-\phi_{11} \phi_{12} n_{2}+(1-\nu) s_{2}(\Delta \phi)^{c}\right\} \\
&= \frac{1+\nu}{E}\left\{\left(\phi_{12}^{2}-\phi_{11} \phi_{22}\right) n_{1}+(1-\nu)\left(s_{1} \Delta \phi+s_{2}(\Delta \phi)^{c}\right)\right\} .
\end{aligned}
$$

We now compute the first component of the $J$-integral by using (2.22) and (2.34):

$$
\begin{aligned}
J_{\Lambda}^{1} & =\int_{\Lambda}\left(W n_{1}-\vec{s} \cdot D_{1} u\right) \\
& =\frac{1-\nu^{2}}{2 E} \int_{\Lambda}\left\{(\triangle \phi)^{2} n_{1}-2 \vec{s} \cdot \vec{\Phi}\right\}
\end{aligned}
$$

where

$$
\vec{\Phi}=\left(\triangle \phi,(\triangle \phi)^{c}\right)
$$

Similarly,

$$
\begin{aligned}
\vec{s} \cdot D_{2} u & =s_{1} D_{2} u_{1}+s_{2} \epsilon_{22} \\
& =\frac{1+\nu}{E}\left\{s_{1}\left[-\phi_{12}-(1-\nu)(\triangle \phi)^{c}\right]+s_{2}\left[-\phi_{22}-(1-\nu) \triangle \phi\right]\right\} \\
& =\frac{1+\nu}{E}\left\{\left(\phi_{12}^{2}-\phi_{11} \phi_{22}\right) n_{2}+(1-\nu)\left(-s_{1}(\triangle \phi)^{c}+s_{2} \triangle \phi\right)\right\}
\end{aligned}
$$

Hence

$$
\begin{aligned}
J_{\Lambda}^{2} & =\int_{\Lambda}\left(W n_{2}-\vec{s} \cdot D_{2} u\right) \\
& =\frac{1-\nu^{2}}{2 E} \int_{\Lambda}\left\{(\triangle \phi)^{2} n_{2}-2 \vec{s} \cdot \vec{\Phi}^{c}\right\}
\end{aligned}
$$

where

$$
\vec{\Phi}^{c}=\left(-(\triangle \phi)^{c}, \triangle \phi\right)
$$


Having computed $\vec{J}$ we can now state the free boundary problem in terms of $\phi$ :

Problem (P)

Let $\Omega$ be a domain in $\mathbb{R}^{2}$ with smooth (say $C^{4+\alpha}$ ) boundary and let $\Gamma_{0}$ be a $C^{4+\alpha}$ curve in $\Omega$ with one end-point on $\partial \Omega$ and the other, $X_{0}$, in $\Omega$. Given continuous functions $f, g$ on $\partial \Omega$ and positive constants $\gamma_{0}, v_{\infty}$, find functions $\phi(x, y, t), X(t)=(x(t), y(t))$ for $t \geq 0$ such that

$$
\triangle^{2} \phi=0 \text { in } \Omega \backslash \overline{\Gamma(t)}
$$

where

$$
\begin{aligned}
\Gamma(t) & =\Gamma_{0} \cup\{X(s) ; 0 \leq s \leq t\}, \quad X(0)=X_{0}, \\
\phi & =f, \quad \frac{\partial \phi}{\partial n}=g \text { on } \partial \Omega, \\
\phi & =0, \quad \frac{\partial \phi}{\partial n}=0 \text { on } \Gamma(t) \text { (from both sides), }
\end{aligned}
$$

and

$$
\dot{X}(t)=\frac{v(t)}{\gamma_{0}} \frac{v_{\infty}-v(t)}{v_{\infty}+v(t)} \vec{J}(X(t)), v(t)=|\dot{X}(t)|,
$$

where $\vec{J}(X(t))$ is defined by (1.14) and (2.35), (2.38). We observe that if $|\vec{J}(X(t))| \leq \gamma_{0}$, then (2.44) implies that $\dot{X}(t)=0$.

\section{The biharmonic equation in a strip}

Let

$$
\begin{aligned}
& D=\{(x, y) ; \quad x \in \mathbb{R},-a<y<a\} \quad(a>0), \\
& \Gamma_{0}=\{(x, 0) ;-\infty<x<0\}, \quad D_{0}=D \backslash \bar{\Gamma}_{0} .
\end{aligned}
$$

Consider the elliptic problem,

$$
\begin{aligned}
& \triangle^{2} \psi=F \text { in } D_{0} \\
& \psi=\frac{\partial \psi}{\partial n}=0 \text { on } \partial D_{0} .
\end{aligned}
$$

Lemma 3.1. If $F$ is in $L^{\infty}(D)$, then there exists a unique bounded solution $\psi$ of (3.2) in $H_{l o c}^{2}(D)$, and

$$
\|\psi\|_{L^{\infty}(D)} \leq C\|F\|_{L^{\infty}(D)}
$$

where $C$ is a constant independent of $F$.

Proof. Consider first the case when $F$ has compact support, and introduce the functional

$$
J(v)=\int_{D_{0}}|\Delta v|^{2}-2 \int_{D_{0}} F v, v \in H_{0}^{2}\left(D_{0}\right) .
$$

One can easily show that $J(v)$ attains a minimum, and that any minimizer $\psi$ is a solution of (3.2); we use here the fact that [1]

$$
\|v\|_{H^{2}(D)}^{2} \leq C \int_{D}|\Delta v|^{2}
$$


Observe that $J(\psi)=\min J(v) \leq J(0)=0$, so that

$$
\int_{D}|\triangle \psi|^{2} \leq 2 \int_{D} F \psi
$$

To prove uniqueness suppose that $\psi_{1}$ is another bounded solution and set $v=\psi-\psi_{1}$. Then $\Delta v=0$ in $D_{0}$. By a Phragmén-Lindelöf type theorem of Lax [9] (see also [6]) $v$ decays exponentially as $x \rightarrow \pm \infty$. By elliptic estimates [2] the same is true for the first three derivatives of $v$. Hence, if we integrate by parts in

$$
\int_{D_{R}} \triangle v \cdot \triangle v \quad\left(D_{R}=D \cap\{|x|<R)\right.
$$

we obtain $\int_{D_{R}} v \triangle^{2} v$ plus boundary integrals (on $|x|=R$ ) which decay exponentially to zero as $R \rightarrow \infty$. It follows that $\int_{D}|\Delta v|^{2}=0$, so that $\psi-\psi_{1} \equiv v \equiv 0$.

The above results are valid also if we replace $H_{0}^{2}\left(D_{0}\right)$ by $H_{0}^{2}(D)$.

Consider next the case of general $F$, and let

$$
F_{j}=F \chi_{j}, \quad \hat{F}_{m}=\sum_{j=-m}^{m-1} F_{j}
$$

where $\chi_{j}=\chi_{j}(x)$ is the characteristic function of $\{j \leq x<j+1\}$. Denote by $\hat{\phi}_{m}$ the bounded solution in $H^{2}(D)$ of

$$
\begin{aligned}
& \triangle^{2} \hat{\phi}_{m}=\hat{F}_{m} \text { in } D, \\
& \hat{\phi}_{m}=\frac{\partial \hat{\phi}_{m}}{\partial n}=0 \text { on } \partial D
\end{aligned}
$$

and by $\phi_{j}$ the corresponding solution for $F_{j}$. Then

$$
\hat{\phi}_{m}=\Sigma_{j=-m}^{m-1} \phi_{j} .
$$

From (3.4), (3.5),

$$
\begin{aligned}
\left\|\phi_{j}\right\|_{H^{2}(D)}^{2} & \leq C \int_{D}\left|\triangle \phi_{j}\right|^{2} \leq 2 C \int_{D} \phi_{j} F_{j} \\
& \leq \frac{1}{2} \int_{D}\left(\phi_{j}\right)^{2}+C M^{2}
\end{aligned}
$$

where

$$
M=\|F\|_{L^{\infty}(D)}
$$

Hence

$$
\left\|\phi_{j}\right\|_{H^{2}(D)} \leq C M \text {. }
$$

Applying the theorem of Lax [9] in half-strips $D \cap\{x>j+1\}$ and $D \cap\{x<j\}$ and using (3.6), we get

$$
\left\|\phi_{j}(\xi, \cdot)\right\|_{H^{2}(D \cap\{x=\xi\})} \leq C M e^{-\lambda|\xi-j|}(\lambda>0) .
$$

The same estimate holds for $j<\xi<j+1$, by (3.6) and elliptic estimates. Summing over $j$ and using also elliptic estimates, we get

$$
\left|D^{j} \hat{\phi}_{m}(x, y)\right| \leq C M \quad(0 \leq j \leq 2) .
$$


Consider now the solution $\psi_{m}$ of (3.2) corresponding to $F=F_{m}$. Since the boundary condition for $\psi_{m}$ are not invariant in $x$ (throughout $D_{0}$ ), the proof of (3.7) cannot be directly extended to $\psi_{m}$. We shall, instead, proceed to estimate the difference

$$
\tilde{\psi}_{m}=\psi_{m}-\hat{\phi}_{m}
$$

Clearly

$$
\begin{aligned}
& \triangle^{2} \tilde{\psi}_{m}=0 \text { in } D \cap\{x>0\} \\
& \tilde{\psi}_{m}=\frac{\partial \tilde{\psi}_{m}}{\partial n}=0 \text { on } \partial D \cap\{x>0\}
\end{aligned}
$$

and, by interior-boundary estimates,

$$
\left\|\tilde{\psi}_{m}\right\|_{C^{2}(\cap\{1<x<2\})} \leq C\left\|\tilde{\psi}_{m}\right\|_{L^{\infty}(D \cap\{0<x<3\})} .
$$

By the theorem of Lax [9] (or [6]) and elliptic estimates, we then have for $x>3$,

$$
\left|D^{j} \tilde{\psi}_{m}(x, y)\right| \leq C\left\|\tilde{\psi}_{m}\right\|_{L^{\infty}(D \cap\{0<x<3\})} e^{-\lambda x}(0 \leq j \leq 2, \lambda>0) .
$$

Recalling (3.7) we conclude that

$$
\left|D^{j} \psi_{m}(x, y)\right| \leq C\left[\left\|\psi_{m}\right\|_{L^{\infty}\left(D_{3}\right)}+M\right] e^{-\lambda x}+C M(0 \leq j \leq 2)
$$

if $x>3$. In the same way we can estimate (3.8) for $x<-3$ ( with a different $\lambda>0$ ).

We shall next estimate $\left\|\psi_{m}\right\|_{L^{\infty}\left(D_{3}\right)}$.

Take $R>3$ and let $\eta \in C^{\infty}(\bar{\Omega})$ be a cutoff function such that

$$
0 \leq \eta \leq 1 \text { in } D, \eta=1 \text { in } D_{2 R}, \eta=0 \text { in } D \backslash D_{3 R}
$$

and

$$
|D \eta| \leq \frac{C}{R},\left|D^{2} \eta\right| \leq \frac{C}{R^{2}}
$$

Substituting $\xi=\eta^{4} \psi_{m}$ into

$$
\int_{D} \triangle \psi_{m} \cdot \triangle \xi=\int_{D} \hat{F}_{m} \xi
$$

we get

$$
\int_{D} \eta^{4}\left(\triangle \psi_{m}\right)^{2}=-\int_{D}\left[2 \triangle \psi_{m} \nabla \eta^{4} \cdot \nabla \psi_{m}+\Delta \psi_{m} \Delta \eta^{4} \cdot \psi_{m}\right]+\int_{D} \hat{F}_{m} \eta^{4} \psi_{m}
$$

writing $\nabla \eta^{4}=4 \eta^{3} \nabla \eta, \triangle \eta^{4}=12 \eta^{2}|\nabla \eta|^{2}+4 \eta^{3} \triangle \eta$ and using the Cauchy-Schwarz inequality, we find, after some simple calculations, that

$$
\int_{D} \eta^{4}\left|\triangle \psi_{m}\right|^{2} \leq \frac{C}{R^{2}} \int_{D_{3 R}}\left(\left|\nabla \psi_{m}\right|^{2}+\psi_{m}^{2}\right)+C M \int_{D} \eta^{4}\left|\psi_{m}\right|
$$

Hence

$$
\int_{D_{2 R}}\left|\Delta \psi_{m}\right|^{2} \leq \frac{C}{R^{2}} \int_{D_{3 R}}\left(\left|\nabla \psi_{m}\right|^{2}+\psi_{m}^{2}\right)+C R^{2} M^{2}
$$


Let $\tilde{\eta}$ be another cutoff function in $C^{\infty}(\bar{\Omega})$ such that

$$
0 \leq \tilde{\eta} \leq 1 \text { in } D, \tilde{\eta}=1 \text { in } D_{R}, \tilde{\eta}=0 \text { in } D \backslash D_{2 R}
$$

and

By (3.4),

$$
|D \tilde{\eta}| \leq \frac{C}{R},\left|D^{2} \tilde{\eta}\right| \leq \frac{C}{R^{2}}
$$

$$
\left\|\psi_{m}\right\|_{H^{2}\left(D_{R}\right)}^{2} \leq C \int_{D}\left|\triangle\left(\tilde{\eta} \psi_{m}\right)\right|^{2} .
$$

Expanding $\triangle\left(\tilde{\eta} \psi_{m}\right)$ and using the Cauchy-Schwarz inequality, we find that

$$
\begin{aligned}
\left\|\psi_{m}\right\|_{H^{2}\left(D_{R}\right)} & \leq C \int_{D_{2 R}}\left|\Delta \psi_{m}\right|^{2}+\frac{C}{R^{2}} \int_{D_{2 R}}\left(\left|\nabla \psi_{m}\right|^{2}+\psi_{m}^{2}\right) \\
& =\frac{C}{R^{2}} \int_{D_{3 R}}\left(\left|\nabla \psi_{m}\right|^{2}+\psi_{m}^{2}\right)+C R^{2} M^{2} \text {, by (3.9). }
\end{aligned}
$$

Choosing $R$ large we then have

$$
\left\|\psi_{m}\right\|_{H^{2}\left(D_{R}\right)}^{2} \leq \frac{2 C}{R^{2}} \int_{D \cap\{R<|x|<3 R\}}\left(\left|\nabla \psi_{m}\right|^{2}+\psi_{m}^{2}\right)+2 C R^{2} M^{2} .
$$

Using (3.8) to estimate the integral on the right-hand side, we obtain

$$
\left\|\psi_{m}\right\|_{H^{2}\left(D_{R}\right)}^{2} \leq \frac{C}{R^{2}}\left\|\psi_{m}\right\|_{L^{\infty}\left(D_{3}\right)}^{2}+C_{R} M^{2} .
$$

Now, by Sobolev's embedding

$$
\left\|\psi_{m}\right\|_{L^{\infty}\left(D_{3}\right)} \leq C\left\|\psi_{m}\right\|_{H^{2}\left(D_{3}\right)}
$$

so that (3.11) yields

$$
\left\|\psi_{m}\right\|_{H^{2}\left(D_{R}\right)}^{2} \leq C_{R} M^{2}
$$

if $R$ is large enough. It follows, in particular, that

$$
\left\|\psi_{m}\right\|_{L^{\infty}\left(D_{3}\right)} \leq C M
$$

Using this in (3.8) we find that

$$
\left|\psi_{m}(x, y)\right| \leq C\|F\|_{L^{\infty}(D)} .
$$

By elliptic estimates we can now take a subsequence $\psi_{m}$ which converges to a solution $\psi$ of (3.2), $\psi$ is in $H_{l o c}^{2}(D)$ and (3.3) holds. The uniqueness of the solution $\psi$ is proved as before.

Lemma 3.2. Consider the elliptic problem:

$$
\begin{aligned}
& \triangle^{2} \psi=0 \text { in } D_{0} \\
& \psi=f, \frac{\partial \psi}{\partial n}=g \text { on } \partial D \\
& \psi=\frac{\partial \psi}{\partial n}=0 \text { on } \Gamma_{0} \text { from both sides. }
\end{aligned}
$$


If the first four derivatives of $f$ and the first three derivatives of $g$ are bounded, then there exists a unique bounded solution of (3.12) in $H_{l o c}^{2}(D)$, and

$$
\|\psi\|_{L^{\infty}(D)} \leq C\left[\|f\|_{C^{4}(\partial D)}+\|g\|_{C^{3}(\partial D)}\right]
$$

where $C$ is a constant independent of $f, g$.

Proof. Choose a function $\psi_{0}$ which satisfies

$$
\psi_{0}=f, \frac{\partial \psi_{0}}{\partial n}=g \text { on } \partial D
$$

and whose first four derivatives are bounded by the right-hand side of (3.13), and apply Lemma 3.1 to $\psi-\psi_{0}$.

Later on we shall also need an extension of Lemma 3.2 to

$$
\begin{aligned}
& \triangle^{2} \psi=0 \text { in } D_{0} \\
& \psi=f, \frac{\partial \psi}{\partial n}=g \text { on } \partial D \\
& \psi=0, \frac{\partial \psi}{\partial n}=h \text { on } \Gamma_{0} \text { from both sides. }
\end{aligned}
$$

Lemma 3.3. Assume that $h$ is uniformly bounded and belongs to $C^{\alpha}\left(\bar{\Gamma}_{0}\right)$, where $\frac{1}{2}<$ $\alpha<1$. If $f, g$ are as in Lemma 3.2 then there exists a unique bounded solution of (3.14) in $H_{l o c}^{2}(D)$, and

$$
\|\psi\|_{L^{\infty}(D)} \leq C\left[\|h\|_{L^{\infty}\left(\Gamma_{0}\right)}+\|f\|_{C^{4}(\partial D)}+\|g\|_{C^{3}(\partial D)}\right]
$$

where $C$ is a constant independent if $h, f, g$.

The proof depends upon the construction of a special solution to

$$
\begin{aligned}
& \triangle^{2} \chi=0 \text { in } \mathbb{R}^{2} \backslash \bar{\Gamma}_{0} \\
& \chi=0, \frac{\partial \chi}{\partial y}=h \text { on } \Gamma_{0} \text { (from both side). }
\end{aligned}
$$

Lemma 3.4. Suppose that $h$ is a bounded function on $\Gamma_{0}$ which belongs to $C^{\alpha}\left(\bar{\Gamma}_{0}\right)$, $0<\alpha<1$. Then there exists a bounded solution $\chi$ of (3.16) such that $\chi \in C^{1}\left(\mathbb{R}^{2}\right)$ and

$$
\|\chi\|_{L^{\infty}(\{|y| \leq R\})} \leq C_{R}\|h\|_{L^{\infty}\left(\Gamma_{0}\right)}
$$

for any $R>0$. If further $\alpha \in\left(\frac{1}{2}, 1\right)$, then $\chi \in H_{l o c}^{2}\left(\mathbb{R}^{2}\right)$.

Proof. The function

$$
w\left(x_{1}, y_{1}\right)=\frac{1}{\pi} \int_{-\infty}^{\infty} \frac{x_{1} h\left(-\eta^{2}\right)}{\left(y_{1}-\eta\right)^{2}+x_{1}^{2}} d \eta
$$

is harmonic in $\left\{x_{1}>0\right\}$, and

$$
w\left(0, y_{1}\right)=h\left(-y_{1}^{2}\right)
$$


By the conformal mapping

$$
z_{1}=z^{\frac{1}{2}}\left(z=x+i y, \quad z_{1}=x_{1}+i y_{1}\right)
$$

we get harmonic function

$$
W(x, y)=w\left(x_{1}, y_{1}\right)
$$

in $\mathbb{R}^{2} \backslash \bar{\Gamma}_{0}$, satisfying the boundary condition

$$
W(x, 0 \pm)=h(x) \text { if } x \leq 0 .
$$

We shall prove that the function

$$
\chi(x, y)=y W(x, y)
$$

satisfies all the assumptions of the lemma.

Clearly $\chi$ is biharmonic in $\mathbb{R}^{2} \backslash \bar{\Gamma}_{0}$ and vanishes on $\Gamma_{0}$. Since $\partial_{y} \chi=W+y \partial_{y} W$, in order to prove that $\partial_{y} \chi=h$ on $\Gamma_{0}$ we only need to show that

$$
y \frac{\partial W}{\partial y} \rightarrow 0 \text { if } y \rightarrow 0, x \leq 0
$$

By (3.18)

$$
|\nabla W| \leq C|\nabla w| r^{-\frac{1}{2}}
$$

where $x=r \cos \theta, y=r \sin \theta$. Since $h \in C^{\alpha}, w \in C^{\alpha}\left(\mathbb{R}^{2}\right)$ and, consequently,

$$
|\nabla w| \leq \frac{C}{x_{1}^{1-\alpha}}
$$

It follows that

$$
|y \nabla W| \leq C \frac{r^{\frac{1}{2}} \sin \theta}{r^{\frac{1-\alpha}{2}} \cos ^{1-\alpha} \frac{\theta}{2}} \leq C r^{\frac{\alpha}{2}} \cos \frac{\theta}{2} \rightarrow 0
$$

if $y \rightarrow 0, x \leq 0$. This establishes $(3.20)$ as well as the fact that $\chi \in C^{1}\left(\mathbb{R}^{2}\right)$ (since $\left.\partial_{x} \chi=y \partial_{x} W\right)$.

The estimate (3.17) follows from the integral representation for $w$. It finally remains to show that if $\alpha>\frac{1}{2}$ then $\chi \in H_{l o c}^{2}\left(\mathbb{R}^{2}\right)$.

In view of the relations

$$
\chi_{x x}=y W_{x x}, \quad \chi_{x y}=y W_{x y}+W_{x}, \quad \chi_{y y}=2 W_{y}+y W_{y y},
$$

all we need to show is that

$$
\nabla W \in L_{l o c}^{2}\left(\mathbb{R}^{2}\right), \text { and } y W_{i j} \in L_{l o c}^{2}\left(\mathbb{R}^{2}\right) .
$$

To prove the first assertion we use (3.22) in (3.21) to get

$$
|\nabla W| \leq C \frac{r^{-\frac{1}{2}}}{x_{1}^{1-\alpha}} \leq C \frac{r^{-1+\frac{\alpha}{2}}}{\cos ^{2(1-\alpha) \frac{\theta}{2}}}
$$


Hence, for any disc $B_{R}=\{r<R\}$,

$$
\int_{B_{R}}|\nabla W|^{2} \leq C \int_{0}^{R} r^{-1+\alpha} d r \int_{-\pi}^{\pi} \frac{d \theta}{\cos ^{2(1-\alpha) \frac{\theta}{2}}}<\infty \text { if } \alpha>\frac{1}{2} .
$$

Next, we have

$$
\left|y W_{i j}\right| \leq C|y|\left[\left|D^{2} w\right| r^{-1}+|\nabla w| r^{-\frac{3}{2}}\right]
$$

and, by elliptic estimates,

$$
\left|D^{2} w\right| \leq \frac{C}{x_{1}^{2-\alpha}}
$$

It follows that

$$
\left|y W_{i j}\right| \leq C \frac{r^{\frac{\alpha-2}{2}}}{\cos ^{1-\alpha} \frac{\theta}{2}}
$$

and

$$
\int_{B_{R}}\left|y W_{i j}\right|^{2} \leq C \int_{0}^{R} r^{-1+\alpha} d r \int_{-\pi}^{\pi} \frac{d \theta}{\cos ^{2(1-\alpha) \frac{\theta}{2}}}<\infty .
$$

Proof of Lemma 3.3. Apply Lemma 3.2 to $\psi-\chi$.

We conclude this section with a brief analysis of the behavior of solutions of (3.12) near $(0,0)$.

Lemma 3.5. Let $\psi(x, y)$ be a solution of (3.12) which belongs to $H_{\text {loc }}^{2}(D)$ and which is an even function in $y$. Then

$$
\frac{\partial^{2} \psi}{\partial y^{2}}(x, 0) \text { is in } C^{\infty}\left(\bar{\Gamma}_{0}\right)
$$

and

$$
\frac{\partial^{3} \psi}{\partial y^{3}}(x, 0)=-G(x)|x|^{-\frac{3}{2}} \text { in } \Gamma_{0}
$$

where $G(x)$ is in $C^{\infty}\left(\bar{\Gamma}_{0}\right)$.

Proof. We use polar coordinates and write $\psi=\psi(r, \theta)$. By [14] $\psi$ has a convergent series expansion about $(0,0)$ in $D_{0} \cap B_{R}$, for any $R>0$ such that $B_{R}$ lies in $D$ :

$$
\psi(r, \theta)=\Sigma_{k=1}^{\infty} r^{\frac{k}{2}+1}\left[a_{k} \cos \left(\frac{k}{2}+1\right) \theta+b_{k} \cos \left(\frac{k}{2}-1\right) \theta\right]
$$

where

$$
\begin{aligned}
& a_{k} \cos \left(\frac{k}{2}+1\right) \pi+b_{k} \cos \left(\frac{k}{2}-1\right) \pi=0 \\
& \left(\frac{k}{2}+1\right) a_{k} \sin \left(\frac{k}{2}+1\right) \pi+\left(\frac{k}{2}-1\right) b_{k} \sin \left(\frac{k}{2}-1\right) \pi=0 .
\end{aligned}
$$

(If $\psi(x, y)$ is not even in $y$ then the expansion includes also sine terms). Note that since $\psi \in H_{l o c}^{2}(D)$, the expansion does not include terms with $k$ smaller than 1 . 
If we compute

$$
\frac{\partial^{2}}{\partial y^{2}}\left[r^{\frac{k}{2} \pm 1} \cos \left(\frac{k}{2} \pm 1\right) \theta\right] \text { at } y=0, x<0
$$

and use (3.26), we find that all terms in $\partial^{2} \psi / \partial y^{2}$ with $k$ odd vanish at $\theta= \pm \pi$. This proves the assertion (3.24). The proof of (3.25) is similar: all the terms in $\partial^{3} \psi / \partial y^{3}$ with $k$ even vanish at $\theta= \pm \pi$.

\section{A travelling wave solution}

In this section we solve a crack propagation problem in the strip $D$; the crack will be given by

$$
\Gamma(t)=\left\{(x, 0) ;-\infty<x<x_{0}(t)\right\}, x_{0}(0)=0,
$$

and the stress function is a bounded function $\phi_{0}$ satisfying:

$$
\begin{aligned}
& \triangle^{2} \phi_{0}=0 \text { in } D \backslash \overline{\Gamma(t)}, \\
& \phi_{0}=M, \frac{\partial \phi_{0}}{\partial n}=0 \text { on } \partial D, \\
& \phi_{0}=0, \frac{\partial \phi_{0}}{\partial n}=0 \text { on } \Gamma(t)
\end{aligned}
$$

here $x_{0}(t)$ is to be determined by (2.44).

We take

$$
\phi_{0}(x, y, t)=U\left(x-v_{0} t, y\right)
$$

where $U$ is the bounded solution in $H_{l o c}^{2}(D)$ to

$$
\begin{aligned}
& \triangle^{2} U=0 \text { in } D \backslash \bar{\Gamma}_{0}, \\
& U=M, \frac{\partial U}{\partial n}=0 \text { on } \partial D, \\
& U=0, \frac{\partial U}{\partial n}=0 \text { on } \Gamma_{0}
\end{aligned}
$$

and $\Gamma_{0}$ is as in (3.1); the existence and uniqueness of $U$ was established in Lemma 3.2. Clearly $\phi_{0}$ satisfies (4.2)-(4.4) and therefore it only remains to show that $v_{0}$ can be chosen so that (2.44) is satisfied.

Physical interpretation of the boundary conditions: It is easy to verify that

$$
\phi_{0} \rightarrow M \text { if } x \rightarrow \infty
$$

and

$$
\phi_{0} \rightarrow-\frac{2 M}{a^{3}}|y|^{3}+\frac{3 M}{a^{2}} y^{2} \text { if } x \rightarrow-\infty .
$$

The traction vector is given by

$$
\vec{s}_{0}=\left(\frac{\partial^{2} \phi_{0}}{\partial y^{2}} n_{1}-\frac{\partial^{2} \phi_{0}}{\partial x \partial y} n_{2}, \frac{\partial^{2} \phi_{0}}{\partial x^{2}} n_{2}-\frac{\partial^{2} \phi_{0}}{\partial x \partial y} n_{1}\right),
$$

and

$$
\vec{s}_{0}(-\infty)=\left(\frac{12 M}{a^{3}}|y|-\frac{6 M}{a^{2}}, 0\right), \vec{s}_{0}(\infty)=0 .
$$

On the other hand $\vec{s}_{0}=0$ on $\partial D$, i.e., there is no traction on $y= \pm a$. Thus the crack propagates due only to force $\vec{s}_{0}(-\infty)$ which is applied at $x=-\infty$.

To determine $v_{0}$ we need first evaluate the $J$-integral at the tip $X_{0}(t)=\left(x_{0}(t), 0\right)$. 
Denote by $\Lambda=\Lambda^{+} \cup \Lambda^{-} \cup \Lambda_{l} \cup \Lambda_{r}$ the path shown in Figure $1 ; \Lambda_{l}$ lies in $\{y=-b\}$ and $\Lambda_{r}$ lies in $\{y=b\}$ :

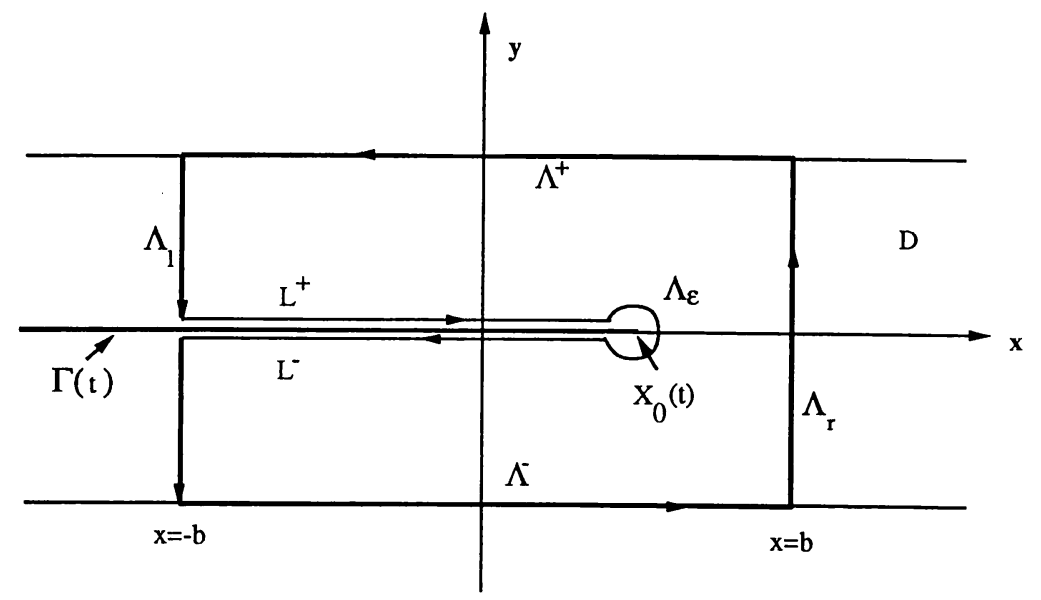

Figure 1

Since $J$-integral is path-independent, we have

$$
J_{1}\left(X_{0}(t)\right)=\frac{1-\nu^{2}}{2 E}\left\{\int_{\Lambda \cup L^{ \pm}}\left(\Delta \phi_{0}\right)^{2} n_{1}-2 \int_{\Lambda \cup L^{ \pm}} \vec{s}_{0} \cdot \vec{\Phi}_{0}\right\}
$$

where $L^{ \pm}$is shown in the figure. Observing that $n_{1}=0$ on $\Lambda^{ \pm}, L^{ \pm}$, and

$$
\vec{s}_{0}=\left(-\frac{\partial}{\partial \tau}\left(\frac{\partial \phi_{0}}{\partial y}\right), \frac{\partial}{\partial \tau}\left(\frac{\partial \phi_{0}}{\partial y}\right)\right)=0 \text { on } \Lambda^{ \pm} \cup L^{ \pm}
$$

we can further write

$$
J_{1}\left(X_{0}(t)\right)=\frac{1-\nu^{2}}{2 E}\left\{\int_{\Lambda_{l} \cup \Lambda_{r}}\left(\Delta \phi_{0}\right)^{2} n_{1}-2 \int_{\Lambda_{l} \cup \Lambda_{r}} \vec{s}_{0} \cdot \vec{\Phi}_{0}\right\} .
$$

From (4.9) and

$$
\vec{\Phi}_{0}=\left(\triangle \phi_{0},\left(\Delta \phi_{0}\right)^{c}\right)
$$

we have

$$
\int_{\Lambda_{l}} \vec{s}_{0} \cdot \vec{\Phi}_{0}=-\int_{\Lambda_{l}} \frac{\partial^{2} \phi_{0}}{\partial y^{2}} \Delta \phi_{0}+\int_{\Lambda_{l}} \frac{\partial^{2} \phi_{0}}{\partial x \partial y}\left(\Delta \phi_{0}\right)^{c} \equiv I_{1}+I_{2}
$$

Integrating by parts in $I_{2}$ and using the Cauchy-Riemann equations, we find that

$$
\begin{aligned}
I_{2} & =-\int_{-a}^{0} \frac{\partial \phi_{0}}{\partial x} \frac{\partial}{\partial y}\left(\Delta \phi_{0}\right)^{c} d y-\int_{0}^{a} \frac{\partial \phi_{0}}{\partial x} \frac{\partial}{\partial y}\left(\Delta \phi_{0}\right)^{c} d y \\
& =-\int_{-a}^{0} \frac{\partial \phi_{0}}{\partial x} \frac{\partial}{\partial x}\left(\triangle \phi_{0}\right) d y-\int_{0}^{a} \frac{\partial \phi_{0}}{\partial x} \frac{\partial}{\partial x}\left(\Delta \phi_{0}\right) d y \rightarrow 0 \quad \text { as }-b \rightarrow-\infty
\end{aligned}
$$

where the last step follows from (4.8). From (4.8) we also get

$$
I_{1} \rightarrow-2 \int_{0}^{a} \lim _{-b \rightarrow-\infty}\left(\frac{\partial^{2} \phi_{0}}{\partial y^{2}}\right)^{2} d y=-2 \int_{0}^{a}\left(-\frac{12 M}{a^{3}} y+\frac{6 M}{a^{3}}\right)^{2} d y=-\frac{24 M^{2}}{a^{3}}
$$




$$
\int_{\Lambda_{l}}\left(\triangle \phi_{0}\right)^{2} n_{1} \rightarrow-\frac{24 M^{2}}{a^{3}} \quad \text { if }-b \rightarrow-\infty .
$$

More simply, from (4.7) we find that

$$
\int_{\Lambda_{r}}\left(\triangle \phi_{0}\right)^{2} n_{1}-2 \int_{\Lambda_{r}} \vec{s}_{0} \vec{\Phi}_{0} \rightarrow 0 \quad \text { as } b \rightarrow \infty .
$$

Collecting these results, we get

$$
J_{1}\left(X_{0}(t)\right)=\frac{12\left(1-\nu^{2}\right) M^{2}}{E a^{3}} .
$$

We next evaluate

$$
J_{2}\left(X_{0}(t)\right)=\frac{1-\nu^{2}}{2 E}\left\{\int_{\Lambda \cup L^{ \pm}}\left(\triangle \phi_{0}\right)^{2} n_{2}-2 \int_{\Lambda \cup L^{ \pm}} \vec{s}_{0} \cdot \vec{\Phi}_{0}^{c}\right\}
$$

here

$$
\vec{\Phi}_{0}^{c}=\left(-\left(\triangle \phi_{0}\right)^{c}, \triangle \phi_{0}\right) .
$$

Since $n_{2}=0$ on $\Lambda_{l} \cup \Lambda_{r}$ and $\vec{s}_{0}=0$ on $\Lambda^{ \pm} \cup L^{ \pm}$, we get

$$
J_{2}\left(X_{0}(t)\right)=\frac{1-\nu^{2}}{2 E}\left\{\int_{\Lambda^{ \pm}}\left(\triangle \phi_{0}\right)^{2} n_{2}+\int_{L^{ \pm}}\left(\triangle \phi_{0}\right)^{2} n_{2}-2 \int_{\Lambda_{l} \cup \Lambda_{r}} \vec{s}_{0} \cdot \vec{\Phi}_{0}^{c}\right\} .
$$

Observing that $\phi_{0}$ is even function in $y$ we deduce that the jump of $\triangle \phi_{0}$ across $L$ is zero and that

$$
\int_{\Lambda^{ \pm}}\left(\triangle \phi_{0}\right)^{2} n_{2}=0
$$

Consequently,

$$
J_{2}\left(X_{0}(t)\right)=-\frac{1-\nu^{2}}{E} \int_{\Lambda_{l} \cup \Lambda_{r}} \vec{s}_{0} \cdot \vec{\Phi}_{0}^{c}
$$

Next, by (4.9),

$$
\int_{\Lambda_{l}} \vec{s}_{0} \cdot \vec{\Phi}_{0}^{c}=\int_{\Lambda_{l}} \frac{\partial^{2} \phi_{0}}{\partial y^{2}}\left(\triangle \phi_{0}\right)^{c}+\int_{\Lambda_{l}} \frac{\partial^{2} \phi_{0}}{\partial x \partial y} \triangle \phi_{0} \equiv \tilde{J}_{1}+\tilde{J}_{2}
$$

and $\tilde{J}_{2} \rightarrow 0$ as $-b \rightarrow-\infty$, by (4.8). As for $\tilde{J}_{1}$, we can integrate by parts and proceed similarly to the analysis of $I_{2}$ in (4.14):

$$
\begin{aligned}
\tilde{J}_{1} & =-\int_{-a}^{0} \frac{\partial \phi_{0}}{\partial y} \frac{\partial}{\partial y}\left(\triangle \phi_{0}\right)^{c} d y-\int_{0}^{a} \frac{\partial \phi_{0}}{\partial y} \frac{\partial}{\partial y}\left(\triangle \phi_{0}\right)^{c} d y \\
& =-\int_{-a}^{0} \frac{\partial \phi_{0}}{\partial y} \frac{\partial}{\partial x}\left(\triangle \phi_{0}\right) d y-\int_{0}^{a} \frac{\partial \phi_{0}}{\partial y} \frac{\partial}{\partial x}\left(\triangle \phi_{0}\right) d y \rightarrow 0 \text { as }-b \rightarrow-\infty
\end{aligned}
$$

by (4.8). Similarly one can show that

$$
\int_{\Lambda_{r}} \vec{s}_{0} \cdot \vec{\Phi}_{0}^{c} \rightarrow 0 \text { if } b \rightarrow \infty
$$


so that altogether

$$
J_{2}\left(X_{0}(t)\right)=0 .
$$

From (2.44), (1.17) and (4.15), (4.17) we see that $\dot{x}_{0}(t) \geq 0$ and

$$
\begin{aligned}
v(t)=\dot{x}_{0}(t) & =v_{\infty} \frac{\left(\left|J_{1}\left(X_{0}(t)\right)\right|-\gamma_{0}\right)_{+}}{\left|J_{1}\left(X_{0}(t)\right)\right|+\gamma_{0}} \\
& =v_{\infty} \frac{\left(\left(12\left(1-\nu^{2}\right) M^{2} / E a^{3}\right)-\gamma_{0}\right)_{+}}{\left(12\left(1-\nu^{2}\right) M^{2} / E a^{3}\right)+\gamma_{0}} \equiv v_{0}
\end{aligned}
$$

It follows that

$$
x_{0}(t)=v_{0} t \text { and } y_{0}(t)=0 \text {. }
$$

We summarize:

Theorem 4.1. There exists a unique bounded travelling wave solution to the special Problem (P) defined by (4.1)-(4.4), where the stress function $\phi_{0}$ is given by (4.5) and the crack propagates along the $x$-axis in accordance with (4.19), where $v_{0}$ is defined by (4.18).

\section{The linearized problem}

We consider a small perturbation of the travelling wave solution constructed in Section 4 , and wish to analyze the resulting linearized problem. We take a small $\epsilon>0$ and define the perturbed boundary conditions:

$$
\begin{gathered}
\phi_{\epsilon}=M+\epsilon, \frac{\partial \phi_{\epsilon}}{\partial n}=0 \text { on } \partial D^{+}=\{y=a\}, \\
\phi_{\epsilon}=M, \frac{\partial \phi_{\epsilon}}{\partial n}=0 \text { on } \partial D^{-}=\{y=-a\}
\end{gathered}
$$

the initial conditions are the same as for the travelling wave solution.

Denote by $\Gamma_{\epsilon}(t)=\Gamma_{0} \cup\left\{X_{\epsilon}(s), 0 \leq s \leq t\right\}$ the perturbed crack. Then $\phi_{\epsilon}$ and $\Gamma_{\epsilon}(t)$ satisfy:

$$
\begin{aligned}
& \triangle^{2} \phi_{\epsilon}=0 \text { in } D \backslash \overline{\Gamma_{\epsilon}(t)}, \\
& \phi_{\epsilon}=0, \frac{\partial \phi_{\epsilon}}{\partial n}=0 \text { on } \Gamma_{\epsilon}(t),
\end{aligned}
$$

and $X_{\epsilon}(t)=\left(x_{\epsilon}(t), y_{\epsilon}(t)\right)$, the tip of $\Gamma_{\epsilon}(t)$, travels according to the law (2.44).

We assume that

$$
\begin{gathered}
\phi_{\epsilon}=\phi_{0}+\epsilon \phi+o(\epsilon), \\
X_{\epsilon}(t)=X_{0}(t)+\epsilon X(t)+o(\epsilon)
\end{gathered}
$$

where $\left(\phi_{0}, X_{0}(t)\right)$ is the solution constructed in Theorem 4.1. Note that

$$
\left.\frac{\partial \phi_{\epsilon}}{\partial \epsilon}\right|_{\epsilon=0}=\phi,\left.\quad \frac{\partial X_{\epsilon}(t)}{\partial \epsilon}\right|_{\epsilon=0}=X(t) \equiv(x(t), y(t)) .
$$

By (5.6) we have

$$
\Gamma_{\epsilon}(t) \rightarrow \Gamma(t) \text { uniformly as } \epsilon \rightarrow 0
$$


where $\Gamma(t)=\left\{(x, 0) ;-\infty<x \leq v_{0} t\right\}$.

Away from $\Gamma_{\epsilon}(t)$ and $\Gamma(t)$,

$$
0=\triangle^{2} \phi_{\epsilon}=\triangle^{2} \phi_{0}+\epsilon \triangle^{2} \phi+o(\epsilon)=\epsilon \triangle^{2} \phi+o(\epsilon)
$$

and, in view of (5.8),

$$
\triangle^{2} \phi=0 \text { in } D \backslash \Gamma(t) .
$$

Clearly, also

$$
\begin{aligned}
& \phi=1, \frac{\partial \phi}{\partial n}=0 \text { on } \partial D^{+} \\
& \phi=0, \frac{\partial \phi}{\partial n}=0 \text { on } \partial D^{-}
\end{aligned}
$$

Next,

$$
0=\phi_{\epsilon}\left(X_{\epsilon}(s), t\right)=\phi_{\epsilon}\left(x_{\epsilon}(s), y_{\epsilon}(s), t\right) \text { for } s<t .
$$

Differentiation of (5.12) with respect to $\epsilon$ yields

$$
0=\frac{\partial \phi_{\epsilon}}{\partial \epsilon}+\frac{\partial \phi_{\epsilon}}{\partial x} \frac{\partial x_{\epsilon}}{\partial \epsilon}+\frac{\partial \phi_{\epsilon}}{\partial y} \frac{\partial y_{\epsilon}}{\partial \epsilon} .
$$

Letting $\epsilon \rightarrow 0$ we get

$$
0=\phi+\frac{\partial \phi_{0}}{\partial x} x(s)+\frac{\partial \phi_{0}}{\partial y} y(s) \text { on } \Gamma(t),
$$

and, since $\nabla \phi_{0}=0$ on $\Gamma(t)$,

$$
\phi=0 \text { on } \Gamma(t) .
$$

Also, from the condition

$$
\nabla \phi_{\epsilon}=0 \text { on } \Gamma_{\epsilon}(t)
$$

we have

$$
0=\frac{\partial \phi_{\epsilon}}{\partial y}\left(x_{\epsilon}(s), y_{\epsilon}(s), t\right) \text { for } s<t .
$$

Differentiating with respect to $\epsilon$, we get

$$
0=\frac{\partial}{\partial y} \frac{\partial \phi_{\epsilon}}{\partial \epsilon}+\frac{\partial^{2} \phi_{\epsilon}}{\partial x \partial y} \frac{\partial x_{\epsilon}}{\partial \epsilon}+\frac{\partial^{2} \phi_{\epsilon}}{\partial y^{2}} \frac{\partial y_{\epsilon}}{\partial \epsilon},
$$

and, therefore, as $\epsilon \rightarrow 0$,

$$
0=\frac{\partial \phi}{\partial y}+\frac{\partial^{2} \phi_{0}}{\partial x \partial y} x(s)+\frac{\partial^{2} \phi_{0}}{\partial y^{2}} y(s) \text { on } \Gamma(t) .
$$

Since $\partial^{2} \phi_{0} / \partial x \partial y=0$ on $\Gamma(t)$, this yields

$$
\frac{\partial \phi}{\partial y}=-\left(\frac{\partial^{2} \phi_{0}}{\partial y^{2}}\right)\left(v_{0} s, 0, t\right) y(s) \text { for } s<t .
$$

Next we derive the motion law for $X(t)$. 
Denote by $\Lambda$ the path shown in the Figure 1. Then

$$
J_{1}\left(X_{\epsilon}(t)\right)=\frac{1-\nu^{2}}{2 E}\left\{\int_{\Lambda}\left(\triangle \phi_{\epsilon}\right)^{2} n_{1}+\int_{\Gamma_{\epsilon, b}^{ \pm}(t)}\left(\triangle \phi_{\epsilon}\right)^{2} n_{1}-2 \int_{\Lambda} \vec{s}_{\epsilon} \cdot \vec{\Phi}_{\epsilon}\right\}
$$

where $\Gamma_{\epsilon, b}(t)=\Gamma_{\epsilon}(t) \cap\{x \geq-b\}$.

Since $n_{1}=0$ on $\partial D$ and $\vec{s}_{\epsilon}=0$ on $\partial D$, we have

$$
J_{1}\left(X_{\epsilon}(t)\right)=\frac{1-\nu^{2}}{2 E}\left\{\int_{\Lambda_{l} \cup \Lambda_{r}}\left(\triangle \phi_{\epsilon}\right)^{2} n_{1}+\int_{\Gamma_{\epsilon, b}^{ \pm}(t)}\left(\triangle \phi_{\epsilon}\right)^{2} n_{1}-2 \int_{\Lambda_{l} \cup \Lambda_{r}} \vec{s}_{\epsilon} \cdot \vec{\Phi}_{\epsilon}\right\} .
$$

We can easily verify (cf. (4.7), (4.8)) that

$$
\phi_{\epsilon} \rightarrow-\frac{\epsilon}{4 a^{3}} y^{3}+\frac{3 \epsilon}{4 a} y+M+\frac{\epsilon}{2} \text { as } x \rightarrow \infty
$$

and

$$
\phi_{\epsilon} \rightarrow\left\{\begin{array}{cc}
-\left[2(M+\epsilon) / a^{3}\right] y^{3}+\left[3(M+\epsilon) / a^{2}\right] y^{2}, & y>0 \\
{\left[2 M / a^{3}\right] y^{3}+\left[3 M / a^{2}\right] y^{2},} & y<0
\end{array} \text { as } x \rightarrow-\infty .\right.
$$

By the method in $\S 4$, as $-b \rightarrow-\infty$,

$$
\begin{aligned}
& \int_{\Lambda_{l}}\left(\Delta \phi_{\epsilon}\right)^{2} n_{1}-2 \int_{\Lambda_{l}} \vec{s}_{\epsilon} \cdot \vec{\Phi}_{\epsilon} \rightarrow \int_{-a}^{a} \lim _{-b \rightarrow-\infty}\left(\frac{\partial^{2} \phi_{\epsilon}}{\partial y^{2}}\right)^{2} d y \\
& =\frac{12 M^{2}}{a^{3}}+\frac{12(M+\epsilon)^{2}}{a^{3}}=\frac{24}{a^{3}} M^{2}+\frac{24}{a^{3}} \epsilon+o(\epsilon) .
\end{aligned}
$$

Similarly, as $b \rightarrow \infty$,

$$
\int_{\Lambda_{r}}\left(\triangle \phi_{\epsilon}\right)^{2} n_{1}-2 \int_{\Lambda_{r}} \vec{s}_{\epsilon} \cdot \vec{\Phi}_{\epsilon} \rightarrow 3 \int_{-a}^{a} \lim _{b \rightarrow \infty}\left(\frac{\partial^{2} \phi_{\epsilon}}{\partial y^{2}}\right)^{2}=o(\epsilon)
$$

Using these relations in (5.16), we get

$$
J_{1}\left(X_{\epsilon}(t)\right)=J_{1}\left(X_{0}(t)\right)+\frac{12\left(1-\nu^{2}\right) M}{E a^{3}} \epsilon+\frac{1-\nu^{2}}{2 E} \int_{\Gamma_{\epsilon, b}(t)^{ \pm}}\left(\triangle \phi_{\epsilon}\right)^{2} n_{1}+o(\epsilon) .
$$

Recall that $\phi_{0}(x, y)=\phi_{0}(x,-y)$ and, consequently, $\partial^{2} \phi_{0} / \partial y^{2}$ and $\Delta \phi_{0}$ are continuous across the crack $\Gamma(t)$. This formally justifies the relation

$$
\triangle \phi_{\epsilon} \rightarrow \triangle \phi_{0} \text { from either side of } \Gamma_{0}(t) .
$$

Along $\Gamma_{\epsilon, b}(t)$ we then have

$$
\begin{aligned}
\left(\triangle \phi_{\epsilon}\right)^{2} n_{1}=-\left(\triangle \phi_{\epsilon}\right)^{2} \dot{y}_{\epsilon}(s) & =-\epsilon\left(\triangle \phi_{\epsilon}\right)^{2}\left(X_{\epsilon}(s), t\right)(\dot{y}(s)+o(1)) \\
& =-\epsilon\left(\triangle \phi_{0}\right)^{2}\left(v_{0} s, 0, t\right)(\dot{y}(s)+o(1)),
\end{aligned}
$$

and we conclude that

$$
\int_{\Gamma_{\epsilon, b}^{ \pm}(t)}\left(\triangle \phi_{\epsilon}\right)^{2} n_{1}=o(\epsilon) .
$$


Substituting this into (5.19) we find that

$$
J_{1}\left(X_{\epsilon}(t)\right)=J_{1}\left(X_{0}(t)\right)+\frac{12\left(1-\nu^{2}\right) M}{E a^{3}} \epsilon+o(\epsilon) .
$$

We next evaluate $J_{2}\left(X_{\epsilon}(t)\right)$, which we can write in the form

$$
J_{2}\left(X_{\epsilon}(t)\right)=\frac{1-\nu^{2}}{2 E}\left\{\int_{\Lambda}\left(\Delta \phi_{\epsilon}\right)^{2} n_{2}+\int_{\Gamma_{\epsilon, b}^{ \pm}(t)}\left(\Delta \phi_{\epsilon}\right)^{2} n_{2}-2 \int_{\Lambda} \vec{s}_{\epsilon} \cdot \vec{\Phi}_{\epsilon}^{c}\right\} .
$$

We can write

$$
\begin{aligned}
\vec{s}_{\epsilon} & =\vec{s}_{0}+\epsilon \vec{s}+o(\epsilon), \\
\vec{\Phi}_{\epsilon}^{c} & =\vec{\Phi}_{0}^{c}+\epsilon \vec{\Phi}^{c}+o(\epsilon)
\end{aligned}
$$

where (cf. (4.9))

$$
\begin{gathered}
\vec{s}_{0}=\left(\frac{\partial^{2} \phi_{0}}{\partial y^{2}} n_{1}-\frac{\partial^{2} \phi_{0}}{\partial x \partial y} n_{2}, \frac{\partial^{2} \phi_{0}}{\partial x^{2}} n_{2}-\frac{\partial^{2} \phi_{0}}{\partial x \partial y} n_{1}\right) \\
\vec{s}=\left(\frac{\partial^{2} \phi}{\partial y^{2}} n_{1}-\frac{\partial^{2} \phi}{\partial x \partial y} n_{2}, \frac{\partial^{2} \phi}{\partial x^{2}} n_{2}-\frac{\partial^{2} \phi}{\partial x \partial y} n_{1}\right)
\end{gathered}
$$

and

$$
\begin{gathered}
\vec{\Phi}_{0}^{c}=\left(-\left(\triangle \phi_{0}\right)^{c}, \triangle \phi_{0}\right), \\
\vec{\Phi}^{c}=\left(-(\triangle \phi)^{c}, \triangle \phi\right) .
\end{gathered}
$$

Then we have

$$
\begin{aligned}
J_{2}\left(X_{\epsilon}(t)\right)= & \frac{1-\nu^{2}}{2 E}\left\{\int_{\Lambda}\left(\triangle \phi_{0}\right)^{2} n_{2}+2 \epsilon \int_{\Lambda}\left(\Delta \phi_{0} \cdot \triangle \phi\right) n_{2}-2 \int_{\Lambda} \vec{s}_{0} \cdot \vec{\Phi}_{0}^{c}\right. \\
& \left.-2 \epsilon \int_{\Lambda}\left(\vec{s}_{0} \cdot \vec{\Phi}^{c}+\vec{s} \cdot \vec{\Phi}_{0}^{c}\right)+\int_{\Gamma_{\epsilon, b}^{ \pm}(t)}\left(\triangle \phi_{\epsilon}\right)^{2} n_{2}\right\}+o(\epsilon) .
\end{aligned}
$$

Denote by $\left[\left(\triangle \phi_{\epsilon}\right)^{2}\right]$ the jump of $\left(\triangle \phi_{\epsilon}\right)^{2}$ across $\Gamma_{\epsilon, b}(t)$ ("plus" above $\Gamma_{\epsilon, b}(t)$ and "minus" below $\left.\Gamma_{\epsilon, b}(t)\right)$. Using this convention we can write

$$
\begin{aligned}
\int_{\Gamma_{\epsilon, b}^{ \pm}(t)}\left(\triangle \phi_{\epsilon}\right)^{2} n_{2} & =-\int_{-b / v_{0}}^{t}\left[\left(\triangle \phi_{\epsilon}\right)^{2}\right]\left(x_{\epsilon}(s), y_{\epsilon}(s), t\right) \dot{x}_{\epsilon}(s) d s \\
& =-v_{0} f(\epsilon)(1+o(\epsilon))
\end{aligned}
$$

where

$$
f(\epsilon)=\int_{-b / v_{0}}^{t}\left[\left(\triangle \phi_{\epsilon}\right)^{2}\right]\left(x_{\epsilon}(s), y_{\epsilon}(s), t\right) d s
$$

Since

$$
\left[\triangle \phi_{0}\right]\left(v_{0} s, 0, t\right)=0 \text { and }\left[\frac{\partial}{\partial x} \triangle \phi_{0}\left(v_{0} s, 0, t\right)\right]=0
$$

we have

$$
\left.f^{\prime}(\epsilon)\right|_{\epsilon=0}=\int_{-b / v_{0}}^{t} 2\left\{\triangle \phi_{0}[\triangle \phi]\left(v_{0} s, 0, t\right)+\Delta \phi_{0}\left[\frac{\partial}{\partial y} \Delta \phi_{0}\right]\left(v_{0} s, 0, t\right) y(s)\right\} d s .
$$


Recalling that $\phi_{0}=\partial \phi_{0} / \partial y=0$ along $\Gamma(t)$, we deduce that

$$
f^{\prime}(0)=2 \int_{-b / v_{0}}^{t} \frac{\partial^{2} \phi_{0}}{\partial y^{2}}\left\{[\triangle \phi]+\left[\frac{\partial^{3} \phi_{0}}{\partial y^{3}}\right] y(s)\right\} d s .
$$

Since $f(0)=0$, we have $f(\epsilon)=f^{\prime}(0) \epsilon+o(\epsilon)$. Using this in (5.29) and substituting the result in (5.28), we arrive at the formula

$$
J_{2}\left(X_{\epsilon}(t)\right)=J_{2}(\phi, y) \epsilon+o(\epsilon)
$$

where

$$
\begin{aligned}
J_{2}(\phi, y)= & \frac{1-\nu^{2}}{E}\left\{\int_{\Lambda}\left(\Delta \phi_{0} \cdot \Delta \phi\right) n_{2}-\int_{\Lambda}\left(\vec{s}_{0} \cdot \vec{\Phi}^{c}+\vec{s} \cdot \vec{\Phi}_{0}^{c}\right)\right\} \\
& -\frac{v_{0}\left(1-\nu^{2}\right)}{E} \int_{-b / v_{0}}^{t} \frac{\partial^{2} \phi_{0}}{\partial y^{2}}\left\{[\Delta \phi]+\left[\frac{\partial^{3} \phi_{0}}{\partial y^{3}}\right] y(s)\right\}\left(v_{0} s, 0, t\right) d s
\end{aligned}
$$

Having computed $J_{1}, J_{2}$ at the tip $X_{\epsilon}(t)$, we now express $\dot{X}_{\epsilon}(t)$ by $(2.44)$ :

$$
\begin{aligned}
& \dot{x}_{\epsilon}(t)=\frac{v_{\epsilon}(t)}{\gamma_{0}} \frac{v_{\infty}-v_{\epsilon}(t)}{v_{\infty}+v_{\epsilon}(t)} J_{1}\left(X_{\epsilon}(t)\right), \\
& \dot{y}_{\epsilon}(t)=\frac{v_{\epsilon}(t)}{\gamma_{0}} \frac{v_{\infty}-v_{\epsilon}(t)}{v_{\infty}+v_{\epsilon}(t)} J_{2}\left(X_{\epsilon}(t)\right) .
\end{aligned}
$$

By $(5.22),(5.32)$,

$$
v_{0}+\epsilon \dot{x}(t)+o(\epsilon)=\frac{v_{\epsilon}(t)}{\gamma_{0}} \frac{v_{\infty}-v_{\epsilon}(t)}{v_{\infty}+v_{\epsilon}(t)} J_{1}\left(X_{0}(t)\right)+\frac{v_{0}}{\gamma_{0}} \frac{v_{\infty}-v_{0}}{v_{\infty}+v_{0}} \frac{12\left(1-\nu^{2}\right) M}{E a^{3}} \epsilon
$$

so that

$$
\epsilon \dot{x}(t)=J_{1}\left(X_{0}(t)\right)\left[\frac{v_{\epsilon}}{\gamma_{0}} \frac{v_{\infty}-v_{\epsilon}}{v_{\infty}+v_{\epsilon}}-\frac{v_{0}}{J_{1}}\right]+\frac{v_{0}}{\gamma_{0}} \frac{v_{\infty}-v_{0}}{v_{\infty}+v_{0}} \frac{J_{1}\left(X_{0}(t)\right)}{M} \epsilon+o(\epsilon)
$$

where (4.15) was used. By (2.44)

$$
J_{1}\left(X_{0}(t)\right)=\gamma_{0} \frac{v_{\infty}+v_{0}}{v_{\infty}-v_{0}}
$$

and, therefore,

$$
\epsilon \dot{x}(t)=J_{1}\left(X_{0}(t)\right)\left\{\left.\frac{d}{d \epsilon}\left[\frac{v_{\epsilon}}{\gamma_{0}} \frac{v_{\infty}-v_{\epsilon}}{v_{\infty}+v_{\epsilon}}\right]\right|_{\epsilon=0}\right\} \epsilon+\frac{v_{0}}{M} \epsilon+o(\epsilon) .
$$

Noting that $\left.\left(d v_{\epsilon} / d \epsilon\right)\right|_{\epsilon=0}=\dot{x}(t)$, it easily follows that

$$
\dot{x}(t)=\frac{J_{1}\left(X_{0}(t)\right)}{\gamma_{0}} \frac{\dot{x}(t)}{\left(v_{\infty}+v_{0}\right)^{2}}\left(v_{\infty}^{2}-v_{0}^{2}-2 v_{\infty} v_{0}\right)+\frac{v_{0}}{M} .
$$

Using (5.34) once more, we get

$$
\dot{x}(t)=\frac{\dot{x}(t)}{v_{\infty}^{2}-v_{0}^{2}}\left(v_{\infty}^{2}-v_{0}^{2}-2 v_{\infty} v_{0}\right)+\frac{v_{0}}{M},
$$


or

$$
\dot{x}(t)=\frac{v_{\infty}^{2}-v_{0}^{2}}{2 v_{\infty} v_{0}} .
$$

Since $x(0)=0$ we finally obtain:

$$
x(t)=\frac{v_{\infty}^{2}-v_{0}^{2}}{2 v_{\infty} v_{0}} t
$$

As for $y(t)$, from (5.33) and (5.30) we get

$$
\dot{y}(t)=\frac{v_{0}}{\gamma_{0}} \frac{v_{\infty}-v_{0}}{v_{\infty}+v_{0}} J_{2}(\phi, y)
$$

where $J_{2}(\phi, y)$ is computed from (5.31) and (5.24)-(5.27).

We summarize the formulation of the linearized problem:

Problem (L).

Given positive constants $\gamma_{0}, v_{\infty}, M$ such that

$$
\frac{12\left(1-\nu^{2}\right) M^{2}}{E a^{3}}>\gamma_{0}
$$

(i.e., $\dot{x}_{0}(t)>0$; see $(4.18)$ ), find functions $\phi=\phi(x, y, t)$ and $y(t)$ such that

$$
\triangle^{2} \phi=0 \text { in } D \backslash \overline{\Gamma(t)}
$$

where $\Gamma(t)=\left\{(x, 0) ;-\infty<x<v_{0} t\right\}$

$$
\begin{gathered}
\phi=1, \frac{\partial \phi}{\partial n}=0 \text { on } \partial D^{+}, \\
\phi=0, \frac{\partial \phi}{\partial n}=0 \text { on } \partial D^{-}, \\
\phi=0, \frac{\partial \phi}{\partial y}\left(v_{0} s, 0, t\right)=-\left(\frac{\partial^{2} \phi_{0}}{\partial y^{2}}\right)\left(v_{0} s, 0, t\right) y(s) \text { on } \Gamma(t)
\end{gathered}
$$

and

$$
\dot{y}(t)=\frac{v_{0}}{\gamma_{0}} \frac{v_{\infty}-v_{0}}{v_{\infty}+v_{0}} J_{2}(\phi, y), y(0)=0
$$

where $J_{2}(\phi, y)$ is defined by $(5.31)$, and $\left(\phi_{0}, v_{0} t\right)$ is the travelling wave solution constructed in Section 4.

Once the linearized problem is solved, we can compute $x(t)$ from (5.35).

\section{Preliminary analysis of the linearized problem}

We first simplify the functional $J_{2}(\phi, y)$ defined in (5.31). By (5.24), (5.25), $\vec{s}_{0}=\vec{s}=0$ on $\partial D$ so that

$$
\int_{\Lambda}\left(\vec{s}_{0} \cdot \vec{\Phi}^{c}+\vec{s} \cdot \vec{\Phi}_{0}^{c}\right)=\int_{\Lambda_{l} \cup \Lambda_{r}}\left(\vec{s}_{0} \cdot \vec{\Phi}^{c}+\vec{s} \cdot \vec{\Phi}_{0}^{c}\right) .
$$


By (5.24), (5.26),

$$
\int_{\Lambda_{l}} \vec{s}_{0} \cdot \vec{\Phi}^{c}=\int_{-a}^{0}\left[\frac{\partial^{2} \phi_{0}}{\partial y^{2}}(-b, y)(\Delta \phi)^{c}(-b, y)+\frac{\partial^{2} \phi_{0}}{\partial x \partial y}(-b, y) \Delta \phi(-b, y)\right] d y .
$$

Integrating by parts,

$$
\begin{aligned}
\int_{-a}^{a} \frac{\partial^{2} \phi_{0}}{\partial y^{2}}(\triangle \phi)^{c} d y & =\int_{-a}^{0} \frac{\partial^{2} \phi_{0}}{\partial y^{2}}(\Delta \phi)^{c} d y+\int_{0}^{a} \frac{\partial^{2} \phi_{0}}{\partial y^{2}}(\triangle \phi)^{c} d y \\
& =-\int_{-a}^{0} \frac{\partial \phi_{0}}{\partial y} \frac{\partial}{\partial y}(\triangle \phi)^{c} d y-\int_{0}^{a} \frac{\partial \phi_{0}}{\partial y} \frac{\partial}{\partial y}(\triangle \phi)^{c} d y \\
& =-\int_{-a}^{0} \frac{\partial \phi_{0}}{\partial y} \frac{\partial}{\partial x} \Delta \phi d y-\int_{0}^{a} \frac{\partial \phi_{0}}{\partial y} \frac{\partial}{\partial x} \Delta \phi d y
\end{aligned}
$$

by the Cauchy-Riemann equations.

Also, by integration by parts,

$$
\int_{-a}^{a}\left(\frac{\partial^{2} \phi_{0}}{\partial x \partial y} \Delta \phi\right)(-b, y) d y=-\int_{-a}^{0} \frac{\partial \phi_{0}}{\partial x} \frac{\partial}{\partial y} \Delta \phi d y-\int_{0}^{a} \frac{\partial \phi_{0}}{\partial x} \frac{\partial}{\partial y} \Delta \phi d y .
$$

Introducing the notation

$$
\nabla^{c}=\left(\frac{\partial}{\partial y}, \frac{\partial}{\partial x}\right)
$$

we get

$$
\int_{\Lambda_{l}} \vec{s}_{0} \cdot \vec{\Phi}^{c}=\int_{\Lambda_{l}}\left(\nabla^{c} \phi_{0} \cdot \nabla \triangle \phi\right) n_{1}
$$

where the right-hand side is defined by

$$
\int_{\Lambda_{l}}\left(\nabla^{c} \phi_{0} \cdot \nabla \Delta \phi\right) n_{1}=\int_{-a}^{0}\left(\nabla^{c} \phi_{0} \cdot \nabla \Delta \phi\right) n_{1}+\int_{0}^{a}\left(\nabla^{c} \phi_{0} \cdot \nabla \Delta \phi\right) n_{1}
$$

and $\left(n_{1}, n_{2}\right)$ is the outward normal.

Similarly,

$$
\int_{\Lambda_{l}} \vec{s} \cdot \vec{\Phi}_{0}^{c}=\int_{\Lambda_{l}}\left(\nabla^{c} \phi \cdot \nabla \triangle \phi_{0}\right) n_{1}
$$

and

$$
\int_{\Lambda_{r}}\left(\vec{s}_{0} \cdot \vec{\Phi}^{c}+\vec{s} \cdot \vec{\Phi}_{0}^{c}\right)=\int_{\Lambda_{r}}\left(\nabla^{c} \phi_{0} \cdot \nabla \Delta \phi+\nabla^{c} \phi \cdot \nabla \triangle \phi_{0}\right) n_{1} .
$$

Using all this in (6.1) and substituting the result into (5.31), we obtain

$$
\begin{gathered}
J_{2}(\phi, y)=\frac{1-\nu^{2}}{E}\left\{\int_{\Lambda}\left(\Delta \phi_{0} \cdot \Delta \phi\right) n_{2}-\int_{\Lambda_{l} \cup \Lambda_{r}}\left(\nabla^{c} \phi_{0} \cdot \nabla \Delta \phi+\nabla^{c} \phi \cdot \nabla \triangle \phi_{0}\right) n_{1}\right\} \\
-\frac{v_{0}\left(1-\nu^{2}\right)}{E} \int_{-b / v_{0}}^{t} \frac{\partial^{2} \phi_{0}}{\partial y^{2}}\left\{[\triangle \phi]+\left[\frac{\partial^{3} \phi_{0}}{\partial y^{3}}\right] y(s)\right\}\left(v_{0} s, 0, t\right) d s .
\end{gathered}
$$

Since $\nabla^{c} \phi_{0}=\nabla \phi=0$ on $\partial D$, we can also write

$$
\begin{array}{r}
J_{2}(\phi, y)=\frac{1-\nu^{2}}{E}\left\{\int_{\Lambda}\left(\Delta \phi_{0} \cdot \Delta \phi\right) n_{2}-\int_{\Lambda}\left(\nabla^{c} \phi_{0} \cdot \nabla \Delta \phi+\nabla^{c} \phi \cdot \nabla \Delta \phi_{0}\right) n_{1}\right\} \\
-\frac{v_{0}\left(1-\nu^{2}\right)}{E} \int_{-b / v_{0}}^{t} \frac{\partial^{2} \phi_{0}}{\partial y^{2}}\left\{[\Delta \phi]+\left[\frac{\partial^{3} \phi_{0}}{\partial y^{3}}\right] y(s)\right\}\left(v_{0} s, 0, t\right) d s .
\end{array}
$$


By Lemma 3.5

$$
\frac{\partial^{2} \phi_{0}}{\partial y^{2}}\left(v_{0} s, 0, t\right) \equiv K(s, t) \text { is } C^{\infty} \text { function in }(s, t)
$$

and

$$
\left[\frac{\partial^{3} \phi_{0}}{\partial y^{3}}\right]\left(v_{0} s, 0, t\right) \equiv-G(s, t)(t-s)^{-\frac{3}{2}}
$$

where $G(s, t)$ is a $C^{\infty}$ function in $(s, t)$. The singularity of $\left[\partial^{3} \phi_{0} / \partial y^{3}\right]\left(v_{0} s, 0, t\right)$ at $s=t$ will cause difficulties when we try to analyze the last integral in (6.5). To overcome these difficulties we shall work with a new function $\psi$, defined by

$$
\psi=\phi+\frac{\partial \phi_{0}}{\partial y} y(t)
$$

Then

$$
\begin{gathered}
\triangle^{2} \psi=0 \text { in } D \backslash \Gamma(t), \\
\psi=1, \quad \frac{\partial \psi}{\partial y}=\frac{\partial^{2} \phi_{0}}{\partial y^{2}} y(t) \text { on } \partial D^{+}, \\
\psi=0, \frac{\partial \psi}{\partial y}=\frac{\partial^{2} \phi_{0}}{\partial y^{2}} y(t) \text { on } \partial D^{-} \\
\psi=0, \quad \frac{\partial \psi}{\partial y}=\frac{\partial^{2} \phi_{0}}{\partial y^{2}}(y(t)-y(s)) \text { on } \Gamma(t),
\end{gathered}
$$

and

$$
\dot{y}(t)=\frac{v_{0}}{\gamma_{0}} \frac{v_{\infty}-v_{0}}{v_{\infty}+v_{0}} \tilde{J}(\psi, y), y(0)=0
$$

where

$$
\begin{gathered}
\tilde{J}(\psi, y)=\frac{1-\nu^{2}}{E}\left\{\int_{\Lambda(t)}\left(\triangle \phi_{0} \cdot \triangle \psi\right) n_{2}-\int_{\Lambda(t)}\left(\nabla^{c} \phi_{0} \cdot \nabla \Delta \psi+\nabla^{c} \psi \cdot \nabla \triangle \phi_{0}\right) n_{1}\right\} \\
-\frac{1-\nu^{2}}{E} y(t)\left\{\int_{\Lambda(t)}\left(\triangle \phi_{0} \cdot \triangle \frac{\partial \phi_{0}}{\partial y}\right) n_{2}-\int_{\Lambda(t)}\left(\nabla^{c} \phi_{0} \cdot \nabla \triangle \frac{\partial \phi_{0}}{\partial y}+\nabla^{c} \frac{\partial \phi_{0}}{\partial y} \cdot \nabla \triangle \phi_{0}\right) n_{1}\right\} \\
-\frac{v_{0}\left(1-\nu^{2}\right)}{E} \int_{-b / v_{0}}^{t} K(s, t)\left\{[\triangle \psi]+G(s, t) \frac{y(t)-y(s)}{(t-s)^{\frac{3}{2}}}\right\} d s ;
\end{gathered}
$$

here $\Lambda(t)$ consists of the four segments

$$
\partial D^{ \pm} \cap\left\{-b \leq x \leq b+v_{0} t\right\}, \quad\{x=-b,|y| \leq a\}, \quad\left\{x=b+v_{0} t,|y| \leq a\right\} .
$$

For any $T>0$ and $0<\alpha<1$, introduce the space

$$
C_{0}^{1+\alpha}[0, T]=\left\{y(s) \in C^{1+\alpha}[0, T], y(0)=0\right\} .
$$

with the usual $C^{1+\alpha}$ norm. 
Take any $y$ in $C_{0}^{1+\alpha}[0, T]$, extend it by zero to $-\infty<s<0$, and define

$$
h(s, t)=\left(\frac{\partial^{2} \phi_{0}}{\partial y^{2}}\right)\left(v_{0} s, 0, t\right)(y(t)-y(s)) .
$$

Then $h$ is Lipschitz continuous in $s \in(-\infty, t]$ and in $C^{1+\alpha}$ for $s \neq 0$. By Lemma 3.3 there exists a unique bounded solution $\psi$ to $(6.9)-(6.12)$. We define $\tilde{J}(\psi, y)$ by $(6.14)$, and a function $\tilde{y}(t)$ by

$$
\frac{d}{d t} \tilde{y}(t)=\frac{v_{0}}{\gamma_{0}} \frac{v_{\infty}-v_{0}}{v_{\infty}+v_{0}} \tilde{J}(\psi, y), \tilde{y}(0)=0 .
$$

Defining a mapping $\mathcal{M}$ by

$$
\tilde{y}=\mathcal{M} y
$$

we shall prove (in the next two sections) that $\mathcal{M}$ has a unique fixed point. This will establish existence and uniqueness of solution to the linearized problem.

\section{Auxiliary estimates}

Take $0<T<1$. For any $\delta \in(0, a)$ we surround the interval $\left\{0 \leq x \leq v_{0} t, y=0\right\}$ by a rectangle

$$
D_{\delta}=\left\{-\delta<x<v_{0} t+\delta,|y|<\delta\right\}
$$

and set

$$
D_{R}^{\delta}=\left(\bar{D} \cap\left\{-R \leq x \leq v_{0} t+R\right\}\right) \backslash\left(D_{\delta} \cup \Gamma(t)\right) .
$$

By Lemma 3.3

$$
\|\psi\|_{L^{\infty}(D)} \leq C\left(1+\|y\|_{C^{0}[0, T]}\right),
$$

and by elliptic estimates we then also have

$$
\|\psi\|_{C^{4}\left(D_{R}^{\delta}\right)} \leq C_{R, \delta}\left(1+\|y\|_{C^{0}[0, T]}\right)
$$

where $C_{R, \delta}$ is a constant independent of $T$. Since $y(0)=0$,

$$
|y(t)| \leq T\|y\|_{C^{1}[0, T]},
$$

so that

$$
\|\psi\|_{C^{4}(\Lambda(t))} \leq C_{R, \delta}\left(1+T\|y\|_{C^{1}[0, T]}\right) .
$$

We next proceed to estimate $[\triangle \psi]\left(v_{0} s, 0, t\right)$ for $-b / v_{0} \leq s \leq t$. For any fixed $t \in[0, T]$, let

$$
\tilde{\psi}=\psi-\chi
$$

where $\chi$ is defined by Lemma 3.4 with $h$ given by (6.15) (as a function of $s$ ). By Lemma 3.4 ,

$$
\|\chi\|_{L^{\infty}(D)} \leq C\|y\|_{C^{\circ}[0, T]}
$$

so that, by (7.1), also

$$
\|\tilde{\psi}\|_{L^{\infty}(D)} \leq C\left(1+\|y\|_{C^{0}[0, T]}\right)
$$


Since $\tilde{\psi} \in H_{l o c}^{2}(D)$ we have [14]:

$$
\tilde{\psi}(r, \theta)=\Sigma_{k=1}^{\infty} r^{\frac{k}{2}+1}\left[a_{k} \cos \left(\frac{k}{2}+1\right) \theta+b_{k} \cos \left(\frac{k}{2}-1\right) \theta+c_{k} \sin \left(\frac{k}{2}-1\right) \theta+d_{k} \sin \left(\frac{k}{2}+1\right) \theta\right]
$$

for $(r, \theta)$ in the disc $B_{\delta}(t)$ with radius $\delta$ and center at the tip $\left(v_{0} t, 0\right)$, where the coefficients $a_{k}, \ldots$ are defined by

$$
a_{k}=\frac{2}{\pi \delta^{\frac{k}{2}+1}} \int_{-\pi}^{\pi} \tilde{\psi}(\delta, \theta) \cos \left(\frac{k}{2}+1\right) \theta d \theta, \ldots ;
$$

$\delta$ is any positive number in the interval $(0, a)$ (so that $B_{\delta}(t) \subset D$ ). It follows that

$$
\left|a_{k}\right| \leq C \delta^{-\left(\frac{k}{2}+1\right)}\|\tilde{\psi}\|_{L^{\infty}(D)}
$$

the same bound holds for $b_{k}, c_{k}, d_{k}$.

Noting that

$$
|\triangle \tilde{\psi}(r, \pi)-\triangle \tilde{\psi}(r,-\pi)| \leq C\left(\left|c_{1}\right|+\left|d_{1}\right|\right) r^{-\frac{1}{2}}+C \Sigma_{k=2}^{\infty} r^{\frac{k}{2}-1}\left(\left|a_{k}\right|+\left|b_{k}\right|+\left|c_{k}\right|+\left|d_{k}\right|\right),
$$

for $r \leq \frac{\delta}{2}$ we get

$$
\left|[\triangle \tilde{\psi}]\left(v_{0} s, 0, t\right)\right| \leq C\|\tilde{\psi}\|_{L^{\infty}(D)}\left(1+\frac{1}{\sqrt{t-s}}\right) \text { if } t-\frac{\delta}{2 v_{0}}<s<t .
$$

Next, by elliptic estimates we get

$$
\left|[\triangle \tilde{\psi}]\left(v_{0} s, 0, t\right)\right| \leq C\left(1+\|\chi\|_{C^{2}}+\|y\|_{C^{0}[0, T]}+\|\tilde{\psi}\|_{L^{\infty}(D)}\right) \text { if }-\frac{b}{v_{0}} \leq s \leq t-\frac{\delta}{2 v_{0}}
$$

where the $C^{2}$ norm of $\chi$ is taken over the intervals $\partial D^{ \pm}, D \cap\{x=-b\}$ and $D \cap\{x=$ $\left.v_{0} t-\delta / 8\right\}$. From the integral representation formula for $\chi$ we easily obtain

$$
\|\chi\|_{C^{2}} \leq C\|y\|_{C^{0}[0, T]} .
$$

Using this in (7.9), combining the result with (7.8), and recalling (7.6), (7.3), we obtain:

Lemma 7.1. The following inequality holds:

$$
\left|[\triangle \psi]\left(v_{0} s, 0, t\right)\right| \leq C\left(1+\sqrt{T}\|y\|_{C^{1}[0, T]}\right)\left(1+\frac{1}{\sqrt{t-s}}\right)+\left|[\triangle \chi]\left(v_{0} s, 0, t\right)\right| .
$$

To estimate $[\triangle \chi]\left(v_{0} s, 0, t\right)$ we shall use the notation of Lemma 3.4, replacing however $\Gamma_{0}$ by $\Gamma(t)$. Thus the origin in the $z$-plane is replaced by $z_{0}=v_{0} t+i \cdot 0$,

$$
z_{1}=\left(z-z_{0}\right)^{\frac{1}{2}}, W(x, y)=w\left(x_{1}, y_{1}\right)
$$

and

$$
w\left(0, y_{1}\right)=\left.W\left(z_{1}^{2}+z_{0}\right)\right|_{x_{1}=0}=h\left(-\frac{1}{v_{0}} y_{1}^{2}+t, t\right) \equiv \tilde{h}\left(y_{1}\right)
$$


where

$$
h(s, t)=\frac{\partial^{2} \phi_{0}}{\partial y^{2}}\left(v_{0} s, 0, t\right)(y(t)-y(s)),-\infty<s<t .
$$

Clearly $h \in C^{1+\alpha}$ except at $s=0$ (since $\dot{y}(0+)$ might not be equal to zero). This means that

$$
w\left(0, y_{1}\right) \in C^{1+\alpha} \text { except at } y_{1}= \pm \sqrt{v_{0} t} .
$$

Lemma 7.2. Let $\hat{w}$ be bounded solution of

$$
\begin{aligned}
& \triangle \hat{w}=0 \text { in } \mathbb{R}^{2} \cap\{x>0\}, \\
& \hat{w}(0, y)=\hat{h}(y), \quad y \in \mathbb{R}
\end{aligned}
$$

where $\hat{h}$ is a bounded $C^{1+\alpha}$ function in $\mathbb{R} \backslash\{0\}$,

$$
\begin{aligned}
& \hat{h}(y)=h_{0}|y| \text { if }|y| \leq 2 R(R \geq 1), \\
& \|\hat{h}\|_{C^{\circ}(\mathbb{R})} \leq C\left|h_{0}\right| R .
\end{aligned}
$$

Then

$$
\left|\hat{w}_{x}(0, y)\right| \leq C\left|h_{0}\right| \log \frac{2 R}{|y|} \text { for }|y| \leq R
$$

where $C$ is a positive constant independent of $h_{0}, R$.

Proof. By scaling we may assume that $R=1$. The function $\zeta=\hat{w}-h_{0}|y|$ satisfies:

$$
\begin{aligned}
& \triangle \zeta=0 \text { in }\left(\mathbb{R}^{2} \cap\{x>0\}\right) \backslash\{y=0\} \\
& \zeta(0, y)=0 \text { in }|y|<2 .
\end{aligned}
$$

Extend $\zeta$ as an odd function into $\{x<0\}$. Then the extended function is harmonic in the strip $\{0<|y|<2\}$ and

$$
\zeta_{y}(x, 0+)-\zeta_{y}(x, 0-)=-2 h_{0} \text { if } x \neq 0 .
$$

By Lemma 4.2 of [5] we deduce that

$$
\left|\zeta_{x}(0, y)\right| \leq C\left|h_{0}\right|(1+|\log | y||)
$$

and the lemma follows.

We now break $\tilde{h}$ into two functions: $\tilde{h}_{0}+\tilde{h}_{1}$ where $\tilde{h}_{0}$ contains the jumps on the derivative of $\tilde{h}$ at $y_{1}= \pm \sqrt{v_{0} t}$ :

$$
\begin{aligned}
\tilde{h}_{0}\left(y_{1}\right)=\tilde{h}^{\prime}\left(\sqrt{v_{0} t}+\right)\left(y_{1}-\sqrt{v_{0} t}\right)_{+}-\tilde{h}^{\prime}\left(\sqrt{v_{0} t}-\right)\left(y_{1}-\sqrt{v_{0} t}\right)_{-} \\
-\tilde{h}^{\prime}\left(-\sqrt{v_{0} t}+\right)\left(y_{1}+\sqrt{v_{0} t}\right)_{+}-\tilde{h}^{\prime}\left(-\sqrt{v_{0} t}-\right)\left(y_{1}+\sqrt{v_{0} t}\right)_{-}
\end{aligned}
$$

if $\left|y_{1}\right| \leq 4 \sqrt{v_{0} T}+\sqrt{b}$ and $\tilde{h}_{0}$ remains bounded at infinity. We can write $w=w_{0}+w_{1}$ where $w_{j}$ is the bounded harmonic function in $\mathbb{R} \backslash\{x \geq 0\}$ with $w_{j}\left(0, y_{1}\right)=\tilde{h}_{j}\left(y_{1}\right)$. Clearly

$$
\left\|\tilde{h}_{0}\right\|_{C^{1+\alpha}\left\{\sqrt{v_{0} t} \leq y_{1} \leq R\right\}} \leq C_{R} \max \left\{\left|\tilde{h}^{\prime}\left( \pm \sqrt{v_{0} t} \pm\right)\right|\right\}
$$


and $\tilde{h}_{1} \in C^{1+\alpha}(\mathbb{R})$.

From the relation

$$
\tilde{h}^{\prime}\left(y_{1}\right)=-\frac{2}{v_{0}} y_{1} h^{\prime}\left(-\frac{1}{v_{0}} y_{1}^{2}+t, t\right)
$$

we deduce that

$$
\left\|\tilde{h}^{\prime}\right\|_{C^{0}\left[-\sqrt{v_{0} t}, \sqrt{v_{0} t}\right]} \leq C \sqrt{T}\|y\|_{C^{1}[0, T]},
$$

since $\left|y_{1}\right| \leq \sqrt{v_{0} T}$. Similarly we can estimate the Hölder coefficient of $\tilde{h}^{\prime}$ and thus get:

$$
\begin{aligned}
\|\tilde{h}\|_{C^{1+\alpha}\left[-\sqrt{v_{0} t}, \sqrt{v_{0} t}\right]} & =\|\tilde{h}\|_{C^{0}\left[-\sqrt{v_{0} t}, \sqrt{v_{0} t}\right]}+\left\|\tilde{h}^{\prime}\right\|_{C^{0}\left[-\sqrt{v_{0} t}, \sqrt{v_{0} t}\right]}+\left\|\tilde{h}^{\prime}\right\|_{C^{\alpha}\left[-\sqrt{v_{0} t}, \sqrt{v_{0} t}\right]} \\
& \leq C \sqrt{T}\|y\|_{C^{1+\alpha}[0, T]}
\end{aligned}
$$

where we used the fact that $\tilde{h}(0)=h(t, t)=0$ and, therefore,

$$
\|\tilde{h}\|_{C^{0}\left[-\sqrt{v_{0} t}, \sqrt{v_{0} t}\right]} \leq T\left\|\tilde{h}^{\prime}\right\|_{C^{0}\left[-\sqrt{v_{0} t}, \sqrt{v_{0} t}\right]} .
$$

For $\left|y_{1}\right|>\sqrt{v_{0} t}, y(s)=0$ so that

$$
\tilde{h}\left(y_{1}\right)=\frac{\partial^{2} \phi_{0}}{\partial y^{2}}\left(-y_{1}^{2}+v_{0} t, 0, t\right) y(t)
$$

Consequently,

$$
\|\tilde{h}\|_{C^{1+\alpha}\left\{\sqrt{v_{0} t} \leq\left|y_{1}\right| \leq R\right\}} \leq C_{R}|y(t)| \leq C_{R} T\|y\|_{C^{1}[0, T]} .
$$

From the form of $\tilde{h}_{0}$ in (7.14) and from (7.16) it follows that $\left|\tilde{h}_{0}^{\prime}\right|$ is bounded by the right hand side of (7.16). Since further $\tilde{h}_{0}$ is piecewise linear, the estimates $(7.16),(7.17)$ hold for the function $\tilde{h}_{1}=\tilde{h}-\tilde{h}_{0}$ and (since $\tilde{h}_{1}$ is continuously differentiable)

$$
\left\|\tilde{h}_{1}\right\|_{C^{1+\alpha}[-R, R]} \leq C_{R} \sqrt{T}\|y\|_{C^{1+\alpha}[0, T]} .
$$

Then, by $C^{1+\alpha}$ estimates for harmonic functions,

$$
\left\|\nabla w_{1}\right\|_{L^{\infty}\left(B_{R}\right)} \leq C R\left[\left\|\tilde{h}_{1}\right\|_{C^{1+\alpha}[-2 R, 2 R]}+\left\|w_{1}\right\|_{L^{\infty}(R)}\right] \leq C_{R} \sqrt{T}\|y\|_{C^{1+\alpha}[0, T]} .
$$

As for $\nabla w_{0}$, it can be estimated by Lemma 7.2. Using also (7.14), (7.16) and (7.17), we find that

$$
\left|\nabla w_{0}\left(0, y_{1}\right)\right| \leq C \sqrt{T}\|y\|_{C^{1+\alpha}[0, T]}\left(1+\left|\log \frac{C}{\left|y_{1}^{2}-v_{0} t\right|}\right|\right) \text {. }
$$

Recalling that $\Delta \chi=2 W_{y}$ and estimating $W_{y}$ from relations (7.11), (7.12) (cf. the proof of Lemma 3.4), we find that

$$
\left|\triangle \chi\left(v_{0} s, 0, t\right)\right| \leq C \sqrt{T}\|y\|_{C^{1+\alpha}[0, T]}\left(1+\left|\log \frac{1}{|s|}\right|\right)
$$

if $-b / v_{0} \leq s \leq t$. Combining this with Lemma 7.1 we conclude:

Lemma 7.3. The following inequality holds:

$$
\left|[\triangle \psi]\left(v_{0} s, 0, t\right)\right| \leq C\left(1+\sqrt{T}\|y\|_{C^{1+\alpha}[0, T]}\right)\left(1+\left|\log \frac{1}{|s|}\right|\right)\left(1+\frac{1}{\sqrt{t-s}}\right)
$$


for $-b / v_{0} \leq s \leq t$, where $C$ is a constant independent of $y$.

We next establish:

Lemma 7.4 The function $\psi(x, y, \cdot)$ is continuously differentiable in $t$, and

$$
\left\|\psi_{t}\right\|_{L^{\infty}(D \times[0, T])} \leq C\|y\|_{C^{1}[0, T]}
$$

where $C$ is independent of $T$.

Proof. Introduce the function $\psi_{1}$ by

$$
\psi_{1}(x, y, t+\Delta t) \equiv \psi\left(x+v_{0} \Delta t, y, t+\Delta t\right) .
$$

From (6.9)-(6.12) we deduce that the function

$$
\psi_{\Delta t}=\frac{1}{\Delta t}\left(\psi_{1}-\psi\right)
$$

satisfies

$$
\begin{aligned}
& \triangle^{2} \psi_{\Delta t}=0 \text { in } D \backslash \overline{\Gamma(t)} \\
& \psi_{\Delta t}=0, \frac{\partial}{\partial y} \psi_{\Delta t}=\frac{1}{\Delta t}\left\{\frac{\partial^{2} \phi_{0}}{\partial y^{2}}(x, \pm a, t)[y(t+\Delta t)-y(t)]\right\} \text { on } \partial D^{ \pm}, \\
& \psi_{\Delta t}=0 \text { on } \Gamma(t) \\
& \frac{\partial}{\partial y} \psi_{\Delta t}=\frac{1}{\Delta t}\left\{\frac{\partial^{2} \phi_{0}}{\partial y^{2}}\left(v_{0} s, 0, t\right)[y(t+\Delta t)-y(s+\Delta t)-y(t)+y(s)]\right\} \text { on } \Gamma(t) .
\end{aligned}
$$

In the boundary conditions we have used the fact that

$$
\phi_{0}\left(v_{0} s-v_{0} \Delta t, 0, t+\Delta t\right)=\phi_{0}\left(v_{0} s, 0, t\right) .
$$

Denote by $\chi_{\Delta t}$ the function $\chi$ introduced in Lemma 3.4 for the boundary data

$$
h\left(v_{0} s\right)=\frac{\partial}{\partial y} \psi_{\Delta t}\left(v_{0} s, 0, t\right)
$$

and let

$$
\tilde{\psi}_{\Delta t}=\psi_{\Delta t}-\chi_{\Delta t}
$$

By Lemma 3.2

$$
\left\|\tilde{\psi}_{\Delta t}\right\|_{L^{\infty}(D)} \leq C\|y\|_{C^{1}[0, T]} .
$$

By elliptic estimates we also have

$$
\left\|\tilde{\psi}_{\Delta t}\right\|_{C^{1+\alpha}(\Omega)} \leq C(\Omega)\|y\|_{C^{1+\alpha}[0, T]} \quad(0<\alpha<1)
$$

for any closed subdomain $\Omega$ of $\bar{D}$ which does not contain the tip $\left(v_{0} t, 0\right)$. Near the tip we have an expansion similar to (7.7) and, by (7.21), the coefficients are bounded by

$$
C \delta^{-\left(\frac{k}{2}+1\right)}\|y\|_{C^{1}[0, T]}
$$


It follows that

$$
\left\|\tilde{\psi}_{\Delta t}\right\|_{C^{1+\alpha}\left(B_{\frac{\delta}{2}}(t)\right)} \leq C\|y\|_{C^{1}[0, T]} \text { if } 0<\alpha<\frac{1}{2} .
$$

From $(7.21),(7.22)$ we see that any sequence $\Delta t \rightarrow 0$ has a subsequence such that

$$
\tilde{\psi}_{\Delta t}(\cdot, t) \rightarrow \tilde{\psi}^{*}(\cdot, t) \text { in } C_{l o c}^{1+\alpha}(\bar{D})
$$

for any $0<\alpha<\frac{1}{2}$, for some function $\tilde{\psi}^{*}$.

On the other hand from the proof of Lemma 3.4 we can deduce that

$$
\chi_{\Delta t} \rightarrow \chi^{*} \text { in } C_{l o c}^{0}(\bar{D})
$$

where $\chi^{*}$ is the bounded solution to

$$
\begin{aligned}
& \triangle^{2} \chi^{*}=0 \text { in } \mathbb{R}^{2} \backslash \overline{\Gamma(t)}, \\
& \chi^{*}=0, \frac{\partial \chi^{*}}{\partial y}=\frac{\partial^{2} \phi_{0}}{\partial y^{2}}\left[y^{\prime}(t)-y^{\prime}(s)\right] \text { on } \Gamma(t) .
\end{aligned}
$$

Combining this fact with (7.23) we find that, for a subsequence $\Delta t \rightarrow 0$,

$$
\psi_{\Delta t}(\cdot, t) \rightarrow \psi^{*}(\cdot, t) \text { uniformly in compact subsets of } \bar{D}
$$

where $\psi^{\prime}$ is a bounded solution to

$$
\begin{aligned}
& \triangle^{2} \psi^{*}=0 \text { in } D \backslash \overline{\Gamma(t)} \\
& \psi^{*}=0, \frac{\partial \psi^{*}}{\partial y}=\left(\frac{\partial^{2} \phi_{0}}{\partial y^{2}}\right)(x, \pm a, t) y^{\prime}(t) \text { on } \partial D^{ \pm} \\
& \psi^{*}=0, \frac{\partial \psi^{*}}{\partial y}=\left(\frac{\partial^{2} \phi_{0}}{\partial y^{2}}\right)\left(v_{0} s, 0, t\right)\left[y^{\prime}(t)-y^{\prime}(s)\right] \text { on } \Gamma(t) .
\end{aligned}
$$

By Lemma $3.3 \psi^{*}$ is the unique bounded solution of (7.25), and

$$
\left\|\psi^{*}(\cdot, t)\right\|_{L^{\infty}(D)} \leq C\|y\|_{C^{1}[0, T]} .
$$

Therefore (7.24) holds for all $\Delta t \rightarrow 0$.

By uniqueness for (7.25) we also deduce that $\psi^{*}(x, y, t)$ is continuous in $t$. Since

$$
\psi^{*}=v_{0} \psi_{x}+\psi_{t} \text { in } D
$$

we further conclude that

$$
\left\|v_{0} \psi_{x}+\psi_{t}\right\|_{L^{\infty}(D)} \leq C\|y\|_{C^{1}[0, T]}
$$

Similarly one can show that $\psi_{x}$ satisfies

$$
\begin{aligned}
& \triangle^{2} \psi_{x}=0 \text { in } D \backslash \overline{\Gamma(t)} \\
& \psi_{x}=0, \frac{\partial \psi_{x}}{\partial y}=\left(\frac{\partial^{3} \phi_{0}}{\partial x \partial y^{2}}\right) y(t) \text { on } \partial D^{ \pm} \\
& \psi_{x}=0 \text { on } \Gamma(t) \\
& \frac{\partial \psi_{x}}{\partial y}=\left(\frac{\partial^{3} \phi_{0}}{\partial x \partial y^{2}}\right)[y(t)-y(s)]-\frac{1}{v_{0}}\left(\frac{\partial^{2} \phi_{0}}{\partial y^{2}}\right) y^{\prime}(s) \text { on } \Gamma(t)
\end{aligned}
$$


and $\psi_{x}$ is continuous in $t$; furthermore

$$
\left\|\psi_{x}(\cdot, t)\right\|_{L^{\infty}(D)} \leq C\|y\|_{C^{1}[0, T]} .
$$

Combining this with the results for $\psi^{*}$, the assertion of the lemma follows.

From Lemma 7.4 and elliptic estimates it follows that $\psi$ is smooth in $(x, y, t)$ for $(x, y) \in$ $D, 0<t<T$, and

$$
\frac{\partial}{\partial t} \Delta \psi=\triangle \psi_{t}
$$

We conclude this section with one more lemma:

Lemma 7.5. The following estimate holds:

$$
\left|\left[\triangle \psi_{t}\right]\left(v_{0} s, 0, t\right)\right| \leq C\left(1+\|y\|_{C^{1+\alpha}[0, T]}\right)(1+|\log | s||)\left(1+\frac{1}{\sqrt{t-s}}\right)
$$

for $-b / v_{0} \leq s \leq t$, where $C$ is a constant independent of $T$ (Recall that $T \leq 1$.).

Proof. By (6.9)-(6.12) and Lemma 7.4,

$$
\begin{aligned}
& \triangle^{2} \psi_{t}=0 \text { in } D \backslash \overline{\Gamma(t)} \\
& \psi_{t}=0, \frac{\partial \psi_{t}}{\partial y}=\left(\frac{\partial^{3} \phi_{0}}{\partial t \partial y^{2}}\right) y(t)+\left(\frac{\partial^{2} \phi_{0}}{\partial y^{2}}\right) y^{\prime}(t) \text { on } \partial D^{ \pm}, \\
& \psi_{t}=0 \text { on } \Gamma(t) \\
& \frac{\partial \psi_{t}}{\partial y}=\left(\frac{\partial^{3} \phi_{0}}{\partial t \partial y^{2}}\right)[y(t)-y(s)]+\left(\frac{\partial^{2} \phi_{0}}{\partial y^{2}}\right) y^{\prime}(t) \text { on } \Gamma(t) .
\end{aligned}
$$

Considering $t$ as a parameter, we can apply the proof of (7.18) to $\psi_{t}$ since the boundary conditions are Lipschitz continuous. Since however the present function $h$ is

$$
h(s, t)=\left(\frac{\partial^{3} \phi_{0}}{\partial t \partial y^{2}}\right)[y(t)-y(s)]+\left(\frac{\partial^{2} \phi_{0}}{\partial y^{2}}\right) y^{\prime}(t)
$$

and it does not generally vanish at the tip (i.e., $h(t, t) \neq 0$ in general), we do not get as in (7.18) a factor $\sqrt{T}$ in front of

$$
\|y\|_{C^{1+\alpha}[0, T]} \text {. }
$$

\section{Existence and uniqueness}

For any $y \in C^{1+\alpha}[0, T]$ we have defined $(\mathcal{M} y)(t)$ by $(6.17)$. Using Lemma 7.1 we easily find that

$$
|\dot{\mathcal{M}}(y)(t)| \leq C\left(1+\sqrt{T}\|y\|_{C^{1+\alpha}[0, T]}\right) .
$$

We next proceed to estimate the $C^{\alpha}$ norm of $\dot{\mathcal{M}}(y)$. Let $0<t_{1}<t_{2}<T$. By (6.16),

$$
\left|\dot{\mathcal{M}}(y)\left(t_{1}\right)-\dot{\mathcal{M}}(y)\left(t_{2}\right)\right| \leq C\left|\tilde{J}(\psi, y)\left(t_{1}\right)-\tilde{J}(\psi, y)\left(t_{2}\right)\right| \text {. }
$$

Using (7.1), (7.2) we easily find that

$$
\left|\dot{\mathcal{M}}(y)\left(t_{1}\right)-\dot{\mathcal{M}}(y)\left(t_{2}\right)\right| \leq C\left(1+\|y\|_{C^{1}[0, T]}\right)\left|t_{1}-t_{2}\right|+I
$$


where

$$
\begin{aligned}
I & =\mid \int_{-b / v_{0}}^{t_{1}} K\left(t_{1}\right)\left[[\Delta \psi]\left(v_{0} s, t_{1}\right)-G\left(t_{1}\right) \frac{y\left(t_{1}\right)-y(s)}{\left(t_{1}-s\right)^{\frac{3}{2}}}\right] d s \\
& -\int_{-b / v_{0}}^{t_{2}} K\left(t_{2}\right)\left[[\Delta \psi]\left(v_{0} s, t_{2}\right)-G\left(t_{2}\right) \frac{y\left(t_{2}\right)-y(s)}{\left(t_{2}-s\right)^{\frac{3}{2}}}\right] d s \mid
\end{aligned}
$$

and

$$
K\left(t_{j}\right)=K\left(t_{j}, s\right), \quad G\left(t_{j}\right)=G\left(t_{j}, s\right), \quad[\triangle \psi]\left(v_{0} s, t_{j}\right)=[\triangle \psi]\left(v_{0} s, 0, t_{j}\right) .
$$

We can write

$$
I \leq I_{1}+I_{2}+I_{3}
$$

where

$$
\begin{gathered}
I_{1}=\int_{-b / v_{0}}^{t_{1}}\left|K\left(t_{1}\right)[\Delta \psi]\left(v_{0} s, t_{1}\right)-K\left(t_{2}\right)[\Delta \psi]\left(v_{0} s, t_{2}\right)\right| d s \\
I_{2}=\int_{-b / v_{0}}^{t_{1}}\left|K\left(t_{1}\right) G\left(t_{1}\right) \frac{y\left(t_{1}\right)-y(s)}{\left(t_{1}-s\right)^{\frac{3}{2}}}-K\left(t_{2}\right) G\left(t_{2}\right) \frac{y\left(t_{2}\right)-y(s)}{\left(t_{2}-s\right)^{\frac{3}{2}}}\right| d s \\
I_{3}=\int_{t_{1}}^{t_{2}}\left|[\Delta \psi]\left(v_{0} s, t_{2}\right)+G\left(t_{2}\right) \frac{y\left(t_{2}\right)-y(s)}{\left(t_{2}-s\right)^{\frac{3}{2}}}\right| d s
\end{gathered}
$$

Using Lemmas 7.3, 7.5, we easily get

$$
\left|I_{1}\right| \leq C\left(1+\|y\|_{C^{1+\alpha}[0, T]}\right) \sqrt{t_{2}-t_{1}} .
$$

Next,

$$
I_{2}=\int_{-b / v_{0}}^{t_{1}} \int_{0}^{1}\left|\frac{d}{d \tau}\left[K\left(t_{2}+\tau\left(t_{1}-t_{2}\right)\right) G\left(t_{2}+\tau\left(t_{1}-t_{2}\right)\right) \frac{y\left(t_{2}+\tau\left(t_{1}-t_{2}\right)\right)-y(s)}{\left(t_{2}+\tau\left(t_{1}-t_{2}\right)-s\right)^{\frac{3}{2}}}\right]\right| d \tau d s .
$$

Applying the derivative $d / d \tau$ first to $K G$ and then to the quotient, we find that

$$
I_{2} \leq C\|y\|_{C^{1}[0, T]}\left(t_{2}-t_{1}\right)^{\frac{3}{2}}+C\|y\|_{C^{1}[0, T]} \int_{-b / v_{0}}^{t_{1}} \int_{0}^{1} \frac{\left|t_{1}-t_{2}\right|}{\left(t_{2}+\tau\left(t_{1}-t_{2}\right)-s\right)^{\frac{3}{2}}} d \tau d s
$$

so that

$$
I_{2} \leq C\|y\|_{C^{1}[0, T]} \sqrt{t_{2}-t_{1}}
$$

Finally, by Lemma 7.3

$$
\begin{gathered}
I_{3} \leq C\left(1+\|y\|_{C^{1+\alpha}[0, T]}\right) \int_{t_{1}}^{t_{2}}|\log s| \frac{1}{\sqrt{t_{2}-t_{1}}} d s \\
C_{\epsilon}\left(1+\|y\|_{C^{1+\alpha}[0, T]}\right)\left(t_{2}-t_{1}\right)^{\frac{1}{2}-\epsilon}
\end{gathered}
$$

for any $\epsilon>0$.

Combining the estimates on the $I_{j}$ and recalling (8.3), (8.4), we get the Hölder estimate

$$
\left|\dot{\mathcal{M}}(y)\left(t_{1}\right)-\dot{\mathcal{M}}(y)\left(t_{2}\right)\right| \leq C_{\epsilon}\left(1+\|y\|_{C^{1+\alpha}[0, T]}\right)\left|t_{1}-t_{2}\right|^{\frac{1}{2}-\epsilon}
$$


for any $0<\epsilon<\frac{1}{2}$.

Choosing $\alpha \in\left(0, \frac{1}{2}\right)$ we deduce from (8.1) and (8.5) that

$$
\mathcal{M} \text { maps } C_{0}^{1+\alpha}[0, T] \text { into itself. }
$$

We next prove:

Lemma 8.1. If $T$ is small enough then $\mathcal{M}$ is a contraction.

Proof. Let $y_{1}, y_{2}$ be two functions in $C_{0}^{1+\alpha}[0, T]$ and denote by $\psi_{1}$ and $\psi_{2}$ corresponding bounded solutions of (6.9)-(6.12). Let $y=y_{1}-y_{2}, \psi=\psi_{1}-\psi_{2}$. By the proof of (8.1) we get

$$
\|\dot{\mathcal{M}}(y)\|_{C^{0}[0, T]} \leq C \sqrt{T}\|y\|_{C^{1+\alpha}[0, T]} .
$$

From (8.5) with $2 \epsilon=\frac{1}{2}-\alpha$ we also have

$$
\|\dot{\mathcal{M}}(y)\|_{C^{\alpha}[0, T]} \leq C T^{\epsilon}\|y\|_{C^{1+\alpha}[0, T]}
$$

and, since $\mathcal{M}(y)(0)=0$,

$$
\|\mathcal{M}(y)\|_{C^{0}[0, T]} \leq C T\|\dot{\mathcal{M}}(y)\|_{C^{0}[0, T]}
$$

Combining these estimates we see that

$$
\left\|\mathcal{M}\left(y_{1}\right)-\mathcal{M}\left(y_{2}\right)\right\|_{C^{1+\alpha}[0, T]}=\|\mathcal{M}(y)\|_{C^{1+\alpha}[0, T]} \leq C T^{\epsilon}\left\|y_{1}-y_{2}\right\|_{C^{1+\alpha}[0, T]}
$$

and the lemma follows.

Theorem 8.2. There exists a unique bounded solution of (6.9)-(6.14) for all $t>0$, with $y \in C^{1+\alpha}[0, T]$ for all $T>0\left(0<\alpha<\frac{1}{2}\right)$.

Proof. From (8.6) and Lemma 8.1 it follows that $\mathcal{M}$ has a unique fixed point in $C^{1+\alpha}[0, T]$ if $T$ is small enough. A step-by-step argument establishes uniqueness for $0<t<t_{0}$ of solutions with $y \in C^{1+\alpha}\left[0, t_{0}\right]$; here $t_{0}$ is any positive number.

A step-by-step argument can also be used to prove global existence. Indeed, a quick look at the proofs of Lemmas 7.1 and 7.3-7.5 shows that the lemmas remain valid for all $0 \leq t \leq T$ with constants $C$ which depend on $T$. Thus if a solution $y(t)$ is already known to exist for $0 \leq t \leq T_{0}$ and to belong to $C^{1+\alpha}\left[0, T_{0}\right]$, we can extend it to a larger interval, say $0 \leq t \leq T_{0}+\delta$, by repeating the previous existence proof; we only need to slightly change the definition of $\tilde{y}$ in (6.16), by taking

$$
\tilde{y}\left(T_{0}\right)=y\left(T_{0}\right)
$$

the constant $\delta$ depends on $T_{0}$, but it remains uniformly positive for $T_{0}$ is any bounded interval.

Theorem 8.3. The initial velocity of the crack tip is upward; more precisely,

$$
\dot{y}(0+)=\frac{6 \sqrt{3} M}{a^{3}} \rho_{0} \text { where } \rho_{0}=\frac{v_{0}}{\gamma_{0}} \frac{v_{\infty}-v_{0}}{v_{\infty}+v_{0}} \frac{1-\nu^{2}}{E} .
$$


In the remaining part of this section we shall prove:

Lemma 8.4.

$$
|\dot{y}(0+)|=\frac{6 \sqrt{3} M}{a^{3}} \rho_{0} .
$$

In $\S 9$ we shall prove that $\dot{y}(0+)>0$, and this together with (8.8) complete the proof of Theorem 8.3.

Proof of Lemma 8.4. By (6.5) and (5.41) we have

$$
\dot{y}(0+)=\rho_{0}\left\{\int_{\Lambda \cup \Gamma_{b}^{ \pm}(0)}\left(\triangle \phi_{0} \cdot \triangle \phi\right) n_{2}-\int_{\Lambda}\left(\nabla^{c} \phi_{0} \cdot \nabla \Delta \phi+\nabla^{c} \phi \cdot \nabla \triangle \phi_{0}\right) n_{1}\right\}
$$

where $\Gamma_{b}(0)=\{(x, 0) ;-b<x<0\}$. Define a function $\phi^{\tau}$ by

$$
\phi^{\tau}(x, y)=\phi(x,-y)
$$

Then $\phi+\phi^{\tau}=\phi_{0} / M$. By elementary calculation, if we replace $\phi_{0}$ by $\phi^{\tau}$ in the right-hand side of (8.8), then each integral is equal to zero. Hence,

$$
\dot{y}(0+)=M \rho_{0}\left\{\int_{\Lambda}(\triangle \phi)^{2} n_{2}+\int_{\Gamma_{b}(0)}\left[(\triangle \phi)^{2}\right] n_{2}-2 \int_{\Lambda} \vec{s} \cdot \vec{\Phi}^{c}\right\} .
$$

Since the integral

$$
\int_{\gamma}\left\{(\triangle \phi)^{2} n_{2}-2 \vec{s} \cdot \vec{\Phi}^{c}\right\}
$$

along any closed curve $\gamma$ is independent of $\gamma$ provided $\gamma$ does not intersect $\Gamma(0)$, we then obtain

$$
\dot{y}(0+)=M \rho_{0} \lim _{\epsilon \rightarrow 0} \int_{\partial B_{e}}\left\{(\triangle \phi)^{2} n_{2}-2 \vec{s} \cdot \vec{\Phi}^{c}\right\}
$$

where $B_{\epsilon}$ is the disc $\left\{x^{2}+y^{2}<\epsilon^{2}\right\}$ and $\partial B_{\epsilon}$ is its boundary.

To compute the right-hand side of (8.9) we use the representation (7.7) in $B_{\epsilon}$ and the conditions $\phi=\partial_{y} \phi=0$ if $y=0, x<0$. Then we get

$$
\phi=r^{3 / 2}\left[\alpha\left(\cos \frac{3}{2} \theta+3 \cos \frac{1}{2} \theta\right)+\beta\left(\sin \frac{3}{2} \theta+\sin \frac{1}{2} \theta\right)\right]+O\left(r^{2}\right) .
$$

It will be convenient to use complex variables:

$$
\begin{gathered}
z=x+i y=r e^{i \theta} \\
\frac{\partial}{\partial z}=\frac{1}{2}\left(\frac{\partial}{\partial x}-i \frac{\partial}{\partial y}\right), \frac{\partial}{\partial \bar{z}}=\frac{1}{2}\left(\frac{\partial}{\partial x}+i \frac{\partial}{\partial y}\right)
\end{gathered}
$$

so that

$$
\triangle=4 \frac{\partial^{2}}{\partial z \partial \bar{z}}
$$

We can then rewrite $(8.10)$ in the form

$$
\phi=A z^{3 / 2}+\bar{A} \bar{z}^{3 / 2}+B z \bar{z}^{1 / 2}+\bar{B} z^{1 / 2} \bar{z}+O\left(|z|^{2}\right)
$$


where

$$
A=\frac{1}{2}(\alpha-i \beta), \quad B=\frac{1}{2}(3 \alpha-i \beta) .
$$

Also

$$
\frac{\partial \phi}{\partial z}=\frac{3}{2} A z^{1 / 2}+B \bar{z}^{1 / 2}+\frac{1}{2} \bar{B} z^{-1 / 2} \bar{z}+O(|z|), \quad \frac{\partial \phi}{\partial \bar{z}}=\overline{\left(\frac{\partial \phi}{\partial z}\right)}
$$

and

$$
\triangle \phi=2 B \bar{z}^{-1 / 2}+2 \bar{B} z^{-1 / 2}+O(1) .
$$

Hence

$$
\int_{\partial B_{\epsilon}}(\triangle \phi)^{2} n_{2}=4 \int_{\partial B_{\epsilon}}\left(B^{2} \bar{z}^{-1}+\bar{B}^{2} z^{-1}+2|B|^{2}|z|^{-1}\right) n_{2}|d z|+O(\epsilon) .
$$

Using the relations

$$
z=\epsilon e^{i \theta}, \quad n_{2}=\sin \theta=\frac{1}{2 i}\left(e^{i \theta}-e^{-i \theta}\right)
$$

we easily compute

$$
\int_{\partial B_{e}}(\triangle \phi)^{2} n_{2}=2 i\left(B^{2}-\bar{B}^{2}\right) 2 \pi+O(\epsilon)=12 \alpha \beta \pi+O(\epsilon) .
$$

To evaluate the integral with $\vec{s} \cdot \Phi^{c}$ in (8.9) we write

$$
\begin{aligned}
\vec{s} & =\left(\frac{\partial^{2} \phi}{\partial y^{2}} \cos \theta-\frac{\partial^{2} \phi}{\partial x \partial y} \sin \theta, \frac{\partial^{2} \phi}{\partial x^{2}} \sin \theta-\frac{\partial^{2} \phi}{\partial x \partial y} \cos \theta\right) \\
& =\left(\frac{1}{r} \frac{\partial}{\partial \theta}\left(\frac{\partial \phi}{\partial y}\right),-\frac{1}{r} \frac{\partial}{\partial \theta}\left(\frac{\partial \phi}{\partial x}\right)\right) .
\end{aligned}
$$

It follows that

$$
\int_{\partial B_{\epsilon}} \vec{s} \cdot \vec{\Phi}^{c}=-\int_{-\pi}^{\pi}\left\{\frac{\partial}{\partial \theta}\left(\frac{\partial \phi}{\partial y}\right)(\triangle \phi)^{c}+\frac{\partial}{\partial \theta}\left(\frac{\partial \phi}{\partial x}\right) \triangle \phi\right\} d \theta
$$

By integration by parts,

$$
\begin{aligned}
\int_{\partial B_{\epsilon}} \vec{s} \cdot \vec{\Phi}^{c} & =\int_{-\pi}^{\pi}\left\{\frac{\partial \phi}{\partial y} \frac{\partial}{\partial \theta}(\Delta \phi)^{c}+\frac{\partial \phi}{\partial x} \frac{\partial}{\partial \theta} \Delta \phi\right\} d \theta \\
& =\int_{\partial B_{\epsilon}} \frac{\partial \phi}{\partial y} \frac{\partial}{\partial r} \Delta \phi|d z|+\frac{1}{\epsilon} \int_{\partial B_{\epsilon}} \frac{\partial \phi}{\partial x} \frac{\partial}{\partial \theta} \Delta \phi|d z| \equiv I_{1}+I_{2}
\end{aligned}
$$

where we have used the Cauchy-Riemann equations

$$
\frac{\partial}{\partial r} \Delta \phi=\frac{1}{r} \frac{\partial}{\partial \theta}(\Delta \phi)^{c}, \frac{1}{r} \frac{\partial}{\partial \theta} \Delta \phi=-\frac{\partial}{\partial r}(\triangle \phi)^{c} .
$$

Now, by (8.11)

$$
\begin{aligned}
& \frac{\partial \phi}{\partial x}=\frac{\partial \phi}{\partial z}+\frac{\partial \phi}{\partial \bar{z}}=\frac{3}{2}\left(A z^{1 / 2}+\bar{A} \bar{z}^{1 / 2}\right)+B \bar{z}^{1 / 2}+\bar{B} z^{1 / 2}+\frac{1}{2}\left(\bar{B} z^{-1 / 2} \bar{z}+B \bar{z}^{-1 / 2} z\right)+O(|z|) \\
& \frac{\partial \phi}{\partial y}=i\left(\frac{\partial \phi}{\partial z}-\frac{\partial \phi}{\partial \bar{z}}\right)=\frac{3}{2} i\left(A z^{1 / 2}-\bar{A} \bar{z}^{1 / 2}\right)+i\left(B \bar{z}^{1 / 2}-\bar{B} z^{1 / 2}\right)+\frac{i}{2}\left(\bar{B} z^{-1 / 2} \bar{z}-B \bar{z}^{-1 / 2} z\right)+O(|z|)
\end{aligned}
$$


and by (8.12)

$$
\begin{gathered}
\frac{\partial}{\partial r} \Delta \phi=\frac{\partial}{\partial z} \Delta \phi \cdot \frac{\partial z}{\partial r}+\frac{\partial}{\partial \bar{z}} \Delta \phi \cdot \frac{\partial \bar{z}}{\partial r}=-\bar{B} z^{-1} \bar{z}^{-1 / 2}-B \bar{z}^{-1} z^{-1 / 2}+O\left(|z|^{-1}\right), \\
\frac{\partial}{\partial \theta} \triangle \phi=\frac{\partial}{\partial z} \Delta \phi \cdot \frac{\partial z}{\partial \theta}+\frac{\partial}{\partial \bar{z}} \Delta \phi \cdot \frac{\partial \bar{z}}{\partial \theta}=-i \bar{B} z^{-1 / 2}+i B \bar{z}^{-1 / 2}+O(1) .
\end{gathered}
$$

Substituting these expressions into the right-hand side of (8.14) we obtain, after simple calculation,

$$
I_{1}=-9 \alpha \beta \pi+O(\epsilon), \quad I_{2}=3 \alpha \beta \pi+O(\epsilon)
$$

Hence

$$
\int_{\partial B_{\epsilon}} \vec{s} \cdot \vec{\Phi}^{c}=-6 \alpha \beta \pi+O(\epsilon)
$$

and together with (8.13) we get, from (8.9),

$$
\dot{y}(0+)=24 M \rho_{0} \alpha \beta \pi .
$$

To compute $\alpha$ we consider the function $\tilde{\phi}=\phi+\phi^{\tau}$. From (8.10) we deduce the expansion

$$
\begin{aligned}
\tilde{\phi} & =2 r^{3 / 2} \alpha\left(\cos \frac{3}{2} \theta+3 \cos \frac{1}{2} \theta\right)+O\left(r^{2}\right), \\
& =\alpha\left(z^{3 / 2}+\bar{z}^{3 / 2}\right)+3 \alpha\left(z \bar{z}^{1 / 2}+z^{1 / 2} \bar{z}\right)+O\left(|z|^{2}\right) .
\end{aligned}
$$

Since $\tilde{\phi}=\phi_{0} / M$, the computation of the $J$-integral in (4.15) gives

$$
J_{1}=\lim _{\epsilon \rightarrow 0} \frac{1-\nu^{2}}{2 E} \int_{\partial B_{\epsilon}}\left\{(\triangle \tilde{\phi})^{2} n_{1}-2 \overrightarrow{\tilde{s}} \cdot \overrightarrow{\tilde{\Phi}}\right\}=\frac{12\left(1-\nu^{2}\right)}{E a^{3}} .
$$

On the other hand, proceeding as before to evaluate the limit of the integral in (8.16) we find that

$$
\int_{\partial B_{\epsilon}}(\triangle \tilde{\phi})^{2} n_{1} \rightarrow 72 \alpha^{2} \pi, \int_{\partial B_{\epsilon}} \overrightarrow{\tilde{s}} \cdot \overrightarrow{\tilde{\Phi}} \rightarrow-36 \alpha^{2} \pi \text { as } \epsilon \rightarrow 0
$$

so that, by $(8.16)$,

$$
\alpha^{2}=\frac{1}{6 \pi a^{3}}
$$

Next we consider the function $\phi^{*}=\phi-\phi^{\tau}$. We compute the $J$-integral in two ways. First we take the integral along the curve $\Lambda$ shown in Figure 1 and let $b \rightarrow \infty$. We then obtain, analogously to (4.15),

$$
J_{1}=\frac{9\left(1-\nu^{2}\right)}{E a^{3}} .
$$

Next we compute $J_{1}$ from the formula

$$
J_{1}=\lim _{\epsilon \rightarrow 0} \frac{1-\nu^{2}}{2 E} \int_{\partial B_{\epsilon}}\left\{\left(\triangle \phi^{*}\right)^{2} n_{1}-2 \overrightarrow{s^{*}} \cdot \overrightarrow{\Phi^{*}}\right\} .
$$

Noting that in $B_{\epsilon}$

$$
\begin{aligned}
\phi^{*} & =2 \beta r^{3 / 2}\left(\sin \frac{3}{2} \theta+\sin \frac{1}{2} \theta\right)+O\left(r^{5 / 2}\right) \\
& =-i \beta\left(z^{3 / 2}-\bar{z}^{3 / 2}\right)-i \beta\left(z \bar{z}^{1 / 2}-z^{1 / 2} \bar{z}\right)+O\left(|z|^{5 / 2}\right)
\end{aligned}
$$


we obtain

$$
\int_{\partial B_{e}}\left(\triangle \phi^{*}\right)^{2} n_{1} \rightarrow-8 \beta^{2} \pi, \int_{\partial B_{e}} \overrightarrow{s^{*}} \cdot \overrightarrow{\Phi^{*}} \rightarrow-12 \beta^{2} \pi \text { as } \epsilon \rightarrow 0
$$

so that

$$
J_{1}=8 \beta^{2} \pi \frac{1-\nu^{2}}{E}
$$

Comparing with (8.18) we find that

$$
\beta^{2}=\frac{9}{8 \pi a^{3}}
$$

Finally, substituting (8.17) and (8.19) into (8.15), the assertion (8.8) follows.

\section{9. $\dot{\mathbf{y}}(0+)>0$}

For any $-\infty<\lambda<b$, let

$$
D_{b}=D \cap\{x<b\}, \Gamma_{\lambda}=\{(x, 0) ; x<\lambda\} .
$$

Consider the following problem:

$$
\begin{aligned}
& \triangle^{2} \phi=0 \text { in } D_{b} \backslash \bar{\Gamma}_{\lambda} \\
& \phi=1, \phi_{y}=0 \text { on } \partial D_{b} \backslash\{x=b\} \\
& \phi=1, \phi_{x x}=0 \text { on }\{x=b\} \\
& \phi=0, \phi_{y}=0 \text { on } \Gamma_{\lambda} \text { (from both sides). }
\end{aligned}
$$

By the analysis of $\S 3$ one can establish the existence of a unique bounded solution $\phi=\phi_{\lambda, b}$ in $H_{l o c}^{2}\left(D_{b}\right)$ of the system (9.1). Furthermore $\phi_{\lambda, b} \in C^{0}\left(\bar{D}_{b}\right)$ and then also, by a standard argument, $\phi_{\lambda, b}$ is continuous in $(x, y, \lambda, b)$ for $(x, y) \in \bar{D}_{b},-\infty<\lambda<b<\infty$. Since, near the tip $(\lambda, 0)$,

$$
\phi_{\lambda, b}=2 \alpha_{\lambda, b} r^{3 / 2}\left(\cos \frac{3}{2} \theta+3 \cos \frac{1}{2} \theta\right)+O\left(r^{2}\right)
$$

where $(r, \theta)$ are polar coordinates about $(\lambda, 0)$, we conclude that

$$
\alpha_{\lambda, b} \text { is continuous with respect to }(\lambda, b) .
$$

Lemma 9.1. $\alpha_{\lambda, b} \neq 0$ for all $-\infty<\lambda<b<\infty$.

Proof. The proof is based on arguments used in proving Lemma 8.4. Set, for simplicity, $\phi=\phi_{\lambda, b}$ and let $\tilde{\phi}$ be the bounded solution in $H_{l o c}^{2}\left(D_{b}\right)$ of the system which is identical to (9.1) except for the boundary condition on $\{x=b\}$ :

$$
\tilde{\phi}_{x}=0 ; \tilde{\phi}_{x x x}=0 \text { on }\{x=b\} .
$$

As in the case of the $J$-integral one can show that the integral

$$
\int_{\gamma}\left[(\triangle \phi \cdot \triangle \tilde{\phi}) n_{1}-(\vec{s} \cdot \overrightarrow{\tilde{\Phi}}+\overrightarrow{\tilde{s}} \cdot \vec{\Phi})\right]
$$


is equal to zero if $\gamma$ encloses a region in $D$ which does not contain cracks.

Let $\Lambda_{b, c}$ be the curve $\Lambda_{b, c}^{l} \cup \Lambda_{b, c}^{r} \cup \Lambda_{b, c}^{ \pm} \cup L_{c}^{ \pm}$shown in Figure 2 when $L_{c}^{ \pm}$converge to the interval $\{(x, 0) ;-c<x<\lambda\}$.

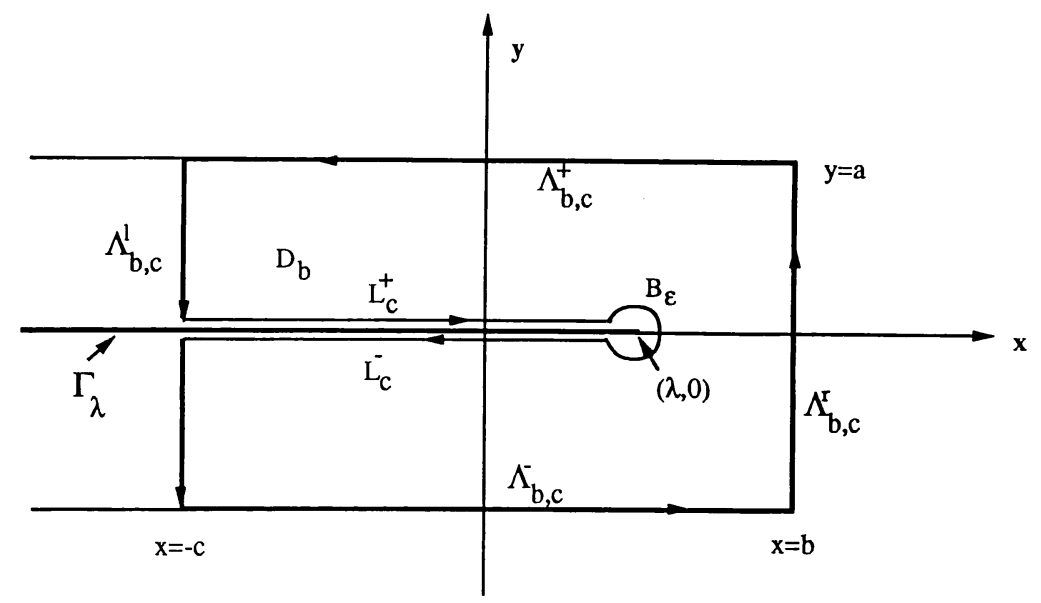

Figure 2

Since $\tilde{\phi}$ is even in $y$, we have

$$
\tilde{\phi}=2 \tilde{\alpha} r^{3 / 2}\left(\cos \frac{3}{2} \theta+3 \cos \frac{1}{2} \theta\right)+O\left(r^{2}\right)
$$

near $(\lambda, 0)$. Proceeding as in the derivation of $(8.17)$, we find that

$$
J \equiv \lim _{\epsilon \rightarrow 0} \int_{\partial B_{\epsilon}(\lambda, 0)}\left\{(\Delta \phi \cdot \Delta \tilde{\phi}) n_{1}-(\vec{s} \cdot \overrightarrow{\tilde{\Phi}}+\overrightarrow{\tilde{s}} \cdot \vec{\Phi})\right\}=72 \pi \alpha_{\lambda, b} \tilde{\alpha} .
$$

On the other hand, by the path independence property of the integral mentioned above, we also have

$$
J=\int_{\Lambda_{b, c}}\left\{(\Delta \phi \cdot \Delta \tilde{\phi}) n_{1}-(\vec{s} \cdot \overrightarrow{\tilde{\Phi}}+\overrightarrow{\tilde{s}} \cdot \vec{\Phi})\right\}
$$

Since $n_{1}=0, \vec{s}=\overrightarrow{\tilde{s}}=0$ on $\Lambda_{b, c}^{ \pm} \cup \Gamma_{\lambda}$, we have

$$
\begin{aligned}
J & =\int_{\Lambda_{b, c}^{l} \cup \Lambda_{b, c}^{r}}\left\{(\Delta \phi \cdot \Delta \tilde{\phi}) n_{1}-(\vec{s} \cdot \overrightarrow{\tilde{\Phi}}+\overrightarrow{\tilde{s}} \cdot \vec{\Phi})\right\} \\
& =-2 \int_{0}^{a}(\Delta \phi \cdot \Delta \tilde{\phi})(-c, y) d y+2 \int_{0}^{a}\left(\frac{\partial^{2} \phi}{\partial y^{2}} \Delta \tilde{\phi}+\frac{\partial^{2} \tilde{\phi}}{\partial y^{2}} \Delta \phi\right)(-c, y) d y \\
& -2 \int_{0}^{a}\left(\frac{\partial^{2} \phi}{\partial x \partial y}(\Delta \tilde{\phi})^{c}+\frac{\partial^{2} \tilde{\phi}}{\partial x \partial y}(\Delta \phi)^{c}\right)(-c, y) d y \\
& +\int_{-a}^{a}(\Delta \phi \cdot \Delta \tilde{\phi})(b, y) d y-\int_{-a}^{a}\left(\frac{\partial^{2} \phi}{\partial y^{2}} \Delta \tilde{\phi}+\frac{\partial^{2} \tilde{\phi}}{\partial y^{2}} \Delta \phi\right)(b, y) d y \\
& +\int_{-a}^{a}\left(\frac{\partial^{2} \phi}{\partial x \partial y}(\Delta \tilde{\phi})^{c}+\frac{\partial^{2} \tilde{\phi}}{\partial x \partial y}(\Delta \phi)^{c}\right)(b, y) d y
\end{aligned}
$$


where we have used the fact that $\phi, \tilde{\phi}$ are even and $(\Delta \phi)^{c},(\Delta \tilde{\phi})^{c}$ are odd with respect to $y$.

Observe that $\partial^{2} \phi / \partial y^{2}=\Delta \phi=0$ at $x=b$. Integrating by parts and using the boundary conditions on $\phi$ and $\tilde{\phi}$, and finally taking $-c \rightarrow-\infty$, we get

$$
\begin{aligned}
J & =2 \int_{0}^{a}\left(\frac{\partial^{2} \phi}{\partial y^{2}} \frac{\partial^{2} \tilde{\phi}}{\partial y^{2}}\right)(-\infty, y) d y-\int_{-a}^{a}\left(\frac{\partial \phi}{\partial x} \frac{\partial}{\partial x} \triangle \tilde{\phi}\right)(b, y) d y-\int_{-a}^{a}\left(\frac{\partial \tilde{\phi}}{\partial x} \frac{\partial}{\partial x} \triangle \phi\right)(b, y) d y \\
& =2 \int_{0}^{a}\left(-\frac{12}{a^{3}} y+\frac{6}{a^{2}}\right)^{2} d y=\frac{24}{a^{3}} .
\end{aligned}
$$

Combining this with (9.4), the assertion of the lemma follows.

Set

$$
\delta=b-\lambda, \phi_{\delta}(x, y)=\phi_{\lambda, b}(x+b, y) .
$$

Lemma 9.2. There holds:

$$
\left\|\phi_{\delta}\right\|_{L^{\infty}} \leq C
$$

where $C$ is a constant independent of $\lambda, \delta$.

Proof. If the assertion is not true then there is a sequence $\delta_{j} \rightarrow 0$ such that

$$
M_{j} \equiv\left\|\phi_{\delta_{j}}\right\| \rightarrow \infty
$$

and, since $\phi_{\delta_{j}}$ is bounded as $x \rightarrow-\infty$, there is a point $\left(x_{j}, y_{j}\right)$ in $D \cap\{x<0\} \cap\{y \geq 0\}$ such that

$$
M_{j}=\phi_{\delta_{j}}\left(x_{j}, y_{j}\right)
$$

(we assume for definiteness that $\sup \left|\phi_{\delta_{j}}\right|=\sup \phi_{\delta_{j}}$ ). Since $\phi_{\delta}(x, y)$ is even in $y$, we can take $y_{j} \geq 0$.

Consider first the case where

$$
x_{j} \rightarrow 0, y_{j} \nrightarrow a, \text { and } \frac{y_{j}}{\left|x_{j}\right|} \rightarrow \infty
$$

and introduce the functions

$$
\phi_{j}(x, y)=\frac{1}{M_{j}} \phi_{\delta_{j}}\left(\left|x_{j}\right| x, y_{j}+\left|x_{j}\right| y\right) .
$$

Then $\phi_{j}(-1,0)=1$ and

$$
\left|\phi_{j}\right| \leq 1 \text { in } D_{j}=\left\{(x, y) ; x<0,-y_{j} /\left|x_{j}\right|<y<\left(a-y_{j}\right) /\left|x_{j}\right|\right\} .
$$

By elliptic estimates, $\phi_{j} \rightarrow \bar{\phi}$ uniformly in compact subsets of $\overline{\mathbb{R}_{-}^{2}}$ where $\mathbb{R}_{-}^{2}=\{(x, y) \in$ $\left.\mathbb{R}^{2}, x<0\right\}$, and $\bar{\phi}$ is the solution to

$$
\begin{aligned}
& \triangle \bar{\phi}=0 \text { in } \mathbb{R}_{-}^{2} \\
& \bar{\phi}=0, \bar{\phi}_{x x}=0 \text { on }\{x=0\} .
\end{aligned}
$$

Also

$$
\bar{\phi}(-1,0)=1 \text {. }
$$


Since $|\bar{\phi}| \leq 1$, by scaling we deduce that

$$
D^{j} \bar{\phi}(x, y)=O\left(r^{-j}\right) \text { if } r \rightarrow \infty .
$$

Using these estimates we find that

$$
0=\int_{l R_{-}^{2} \cap\{r<1 / \epsilon\}} \bar{\phi} \triangle^{2} \bar{\phi}=\int_{l R_{-}^{2} \cap\{r<1 / \epsilon\}}|\triangle \bar{\phi}|^{2}+I_{\epsilon}
$$

where $I_{\epsilon} \rightarrow 0$ if $\epsilon \rightarrow 0$. Consequently $\bar{\phi} \equiv 0$, which is a contradiction to (9.8).

In the case where

$$
y_{j} /\left|x_{j}\right| \text { remain bounded as } x_{j} \rightarrow 0
$$

we work with the sequence

$$
\phi_{j}(x, y)=\frac{1}{M_{j}} \phi_{\delta_{j}}\left(\left|x_{j}\right| x,\left|x_{j}\right| y\right)
$$

Let $\mu=\lim -\delta_{j} /\left|x_{j}\right| \in[-\infty, 0]$. Then $\bar{\phi}$ satisfies (9.7) in $\mathbb{R}_{-}^{2} \backslash \bar{\Gamma}_{\mu}$, and on $\Gamma_{\mu}$ we have $\bar{\phi}=\bar{\phi}_{y}=0$.

If $\mu=0$ or $-\infty$, one can verify, as in the case of (9.6), that $\bar{\phi} \equiv 0$. If $-\infty<\mu<0$, then $\bar{\phi}=O\left(\bar{r}^{3 / 2}\right)$ near $(\mu, 0)$ and, by scaling,

$$
D^{j} \bar{\phi}(x, y)=O\left(\bar{r}^{3 / 2-j}\right) \text { if } \bar{r} \rightarrow 0
$$

where $\bar{r}$ is the distance to $(\mu, 0)$. We then deduce that $\bar{\phi} \equiv 0$ by integrating $\bar{\phi} \triangle^{2} \bar{\phi}$ over $\left(\mathbb{R}_{-}^{2} \backslash \bar{\Gamma}_{\mu}\right) \cap\{\epsilon<\bar{r}<1 / \epsilon\}$ and taking $\epsilon \rightarrow 0$. On the other hand, $\phi_{j}\left(-1, y_{j} /\left|x_{j}\right|\right)=1$. By assumption, we may set $\bar{y}=\lim y_{j} /\left|x_{j}\right| \in[0, \infty)$. Since $\phi_{j} \rightarrow \bar{\phi}$ in $C_{l o c}^{0}\left(\mathbb{R}_{-}^{2}\right)$, we get $\bar{\phi}(-1, \bar{y})=1$, which is a contradiction.

Similarly we can treat the case where $x_{j} \rightarrow 0, y_{j} \rightarrow a$.

Finally, if $\left|x_{j}\right|$ remains uniformly positive, then we use the scaling

$$
\phi_{j}(x, y)=\frac{1}{M_{j}} \phi_{\delta_{j}}\left(x-x_{j}, y\right),
$$

and derive a contradiction in a simpler way.

Lemma 9.3. For $\lambda$ close to $b, \alpha_{\lambda, b}>0$.

Proof. Let

$$
\Phi_{\delta}(x, y)=\phi_{\delta}(\delta x, \delta y) .
$$

By Lemma 9.2 and elliptic estimates,

$$
\Phi_{\delta_{j}} \rightarrow \Phi_{0} \text { uniformly in compact subsets of } \overline{\mathbb{R}_{-}^{2}}
$$

where $\Phi_{0}$ is a bounded solution of

$$
\begin{aligned}
& \triangle^{2} \Phi_{0}=0 \text { in } \mathbb{R}_{-}^{2} \backslash \bar{\Gamma}_{-1} \\
& \Phi_{0}=1, \frac{\partial^{2} \Phi_{0}}{\partial x^{2}}=0 \text { on }\{x=0\} \\
& \Phi_{0}=0, \frac{\partial \Phi_{0}}{\partial y}=0 \text { on } \Gamma_{-1} .
\end{aligned}
$$


As in the proof of Lemma 9.2, one can verify that this bounded solution to (9.9) is unique and, consequently

$$
\Phi_{\delta} \rightarrow \Phi_{0} \text { in } C_{l o c}^{0}\left(\overline{\mathbb{R}_{-}^{2}}\right) .
$$

Observe that near $(-1,0)$,

$$
\Phi_{\delta}=2 \alpha_{\delta} r^{3 / 2}\left(\cos \frac{3}{2} \theta+3 \cos \frac{1}{2} \theta\right)+O\left(r^{2}\right)
$$

where

$$
\alpha_{\delta}=\alpha_{\lambda, b} \delta^{3 / 2}
$$

Hence, in order to complete the proof of Lemma 9.3 it suffices to show that, near the tip $(-1,0)$

$$
\Phi_{0}(x, y)=\alpha_{0} r^{3 / 2}\left(\cos \frac{3}{2} \theta+3 \cos \frac{1}{2} \theta\right)+O\left(r^{2}\right) \text { with } \alpha_{0}>0 .
$$

Let $w$ be the bounded solution to

$$
\begin{aligned}
& \Delta w=0 \text { in } \mathbb{R}_{-}^{2} \backslash \bar{\Gamma}_{-1}, \\
& w=1 \text { on }\{x=0\} \\
& w=0 \text { on } \Gamma_{-1} \text { (from both sides). }
\end{aligned}
$$

By the maximum principle, $0<w<1$. Further, near $(-1,0)$

$$
w=a_{1} r^{1 / 2} \cos \frac{\theta}{2}+O\left(r^{3 / 2}\right) \text { where } a_{1}>0 .
$$

Indeed, this follows from the fact the function $\tilde{w}(z)=w\left(z^{2}-1\right)$ is harmonic in $\operatorname{Re}(z)>0$ and vanishes on $\operatorname{Re}(z)=0$.

Next let $v$ be the bounded solution to

$$
\begin{aligned}
\Delta v & =0 \text { in } \mathbb{R}_{-}^{2} \backslash \bar{\Gamma}_{-1}, \\
v & =0 \text { on }\{x=0\}, \\
v^{ \pm} & =(1+x) w_{y}^{ \pm} \text {on } \Gamma_{-1}^{ \pm}
\end{aligned}
$$

where $v^{ \pm}, w^{ \pm}$are defined in $\left\{y_{<}^{>0}\right\}$. Note that $(1+x) w_{y}^{ \pm}$is in $C^{\alpha}\left(\Gamma_{-1}^{ \pm}\right)$for any $0<\alpha<1 / 2$.

We next introduce the function

$$
\phi_{1}=(1+x) w+y v .
$$

It satisfies:

$$
\begin{gathered}
\triangle^{2} \phi_{1}=0 \text { in } \mathbb{R}_{-}^{2} \backslash \bar{\Gamma}_{-1}, \\
\phi_{1}=1 \text { on }\{x=0\}, \\
\frac{\partial^{2} \phi_{1}}{\partial x^{2}}=2 w_{x} \text { on }\{x=0\}, \\
\phi_{1}=0, \frac{\partial \phi_{1}}{\partial y}=0 \text { on } \Gamma_{-1} .
\end{gathered}
$$


Observe that $\phi_{1} \in C^{1}\left(\mathbb{R}_{-}^{2}\right)$ and $\phi_{1}$ grows linearly at $\infty$ ( since $w$ and $v$ are bounded). Further,

$$
\phi_{1}=a_{1}(1+x)^{3 / 2}+O\left((1+x)^{5 / 2}\right) \text { if } y=0,-1<x<0 .
$$

We note by the maximum principle, that $w_{x}>0$.

To get rid of the $2 w_{x}$ in (9.13) we shall construct two auxiliary functions: $w_{2}$, the bounded solution to

$$
\begin{aligned}
& \triangle w_{2}=0 \text { in } \mathbb{R}_{-}^{2} \backslash \bar{\Gamma}_{-1}, \\
& w_{2}+\frac{\partial w_{2}}{\partial x}+y \frac{\partial w_{2}}{\partial y}=-w_{x} \text { on }\{x=0\} \\
& w_{2}=0 \text { on } \Gamma_{-1} \text { (from both sides) }
\end{aligned}
$$

and $v_{2}$, a linearly bounded solution to

$$
\begin{aligned}
& \triangle v_{2}=0 \text { in } \mathbb{R}_{-}^{2} \backslash \bar{\Gamma}_{-1}, \\
& v_{2}=-y w_{2} \text { on }\{x=0\}, \\
& v_{2}=x(1+x)\left(\frac{\partial w_{2}}{\partial y}\right)^{ \pm} \text {on } \Gamma_{-1}^{ \pm} .
\end{aligned}
$$

We construct $w_{2}$ by solving truncated problems in $\mathbb{R}_{-}^{2} \cap\left\{x^{2}+y^{2}<\rho^{2}\right\}$ with

$$
w_{2}=0 \text { on } x^{2}+y^{2}=\rho^{2},
$$

and observe, by the maximum principle, that

$$
-A<w_{2}<0 \quad\left(A=\sup \left|w_{x}\right|\right) .
$$

We also construct $v_{2}$ by truncations, and use the Phragmén-Lindelöf theorem to deduce that

$$
\left|v_{2}\right|=O(r) \text { as } r \rightarrow \infty .
$$

Using conformal mapping and the maximum principle we prove, as in (9.12), that near the tip $(-1,0)$

$$
w_{2}=a_{2} r^{1 / 2} \cos \frac{\theta}{2}+O\left(r^{3 / 2}\right) \text { where } a_{2}<0 .
$$

Since $w_{2}$ is bounded, $(9.15)$ gives

$$
w_{2}+\left.y \frac{\partial w_{2}}{\partial y}\right|_{x=0}=O\left(\frac{1}{|y|}\right) \text { if }|y| \rightarrow \infty
$$

and, by integration,

$$
\left|w_{2}(0, y)\right| \leq C \frac{\log |y|}{|y|} \text { if }|y| \rightarrow \infty .
$$

Therefore, by the Phragmén-Lindelöf theorem, for any $\epsilon>0$,

$$
\left|w_{2}\right| \leq C \frac{1+r^{\epsilon}}{1+r} \text { in } \mathbb{R}_{-}^{2}
$$


from which we deduce that

$$
\left|x(1+x)\left(\frac{\partial w_{2}}{\partial y}\right)^{ \pm}\right| \leq C\left(1+|x|^{\epsilon}\right) \text { on } \Gamma_{-1}^{ \pm} .
$$

Therefore (again by the Phragmén-Lindelöf theorem), $v_{2}$ is bounded sublinearly:

$$
\left|v_{2}\right| \leq C\left(1+r^{\epsilon}\right) \text { in } \mathbb{R}_{-}^{2} .
$$

Now let

$$
\phi_{2}=x(1+x) w_{2}+y^{2} w_{2}+y v_{2}
$$

Then

$$
\left|\phi_{2}\right| \leq C\left(1+r^{1+\epsilon}\right) \text { in } \mathbb{R}_{-}^{2}
$$

and, as easily seen, $\phi_{2}=O\left(r^{3 / 2}\right)$ near the tip $(-1,0)$ so that

$$
\phi_{2} \in H_{l o c}^{2}\left(\mathbb{R}_{-}^{2}\right) \text {. }
$$

The function $\phi_{2}$ satisfies

$$
\begin{gathered}
\triangle^{2} \phi_{2}=0 \text { in } \mathbb{R}_{-}^{2} \backslash \bar{\Gamma}_{-1}, \\
\phi_{2}=0 \text { on }\{x=0\} \\
\frac{\partial^{2} \phi_{2}}{\partial x^{2}}=-2 w_{x} \text { on }\{x=0\} \\
\phi_{2}=0, \frac{\partial \phi_{2}}{\partial y}=0 \text { on } \Gamma_{-1} .
\end{gathered}
$$

It follows that the function

$$
\hat{\Phi} \equiv \phi_{1}+\phi_{2}
$$

satisfies the system (9.9). Furthermore, using (9.14), (9.17) we find, by restricting the expansion near $(-1,0)$,

$$
\hat{\Phi}=a r^{3 / 2}\left(\cos \frac{3}{2} \theta+3 \cos \frac{1}{2} \theta\right)+O\left(r^{2}\right)
$$

to $\{(x, 0),-1<x<0\}$, that

$$
a=a_{1}-a_{2}>0 .
$$

Thus if we can prove that $\hat{\Phi} \equiv \Phi_{0}$ then assertion (9.11) follows and the proof of Lemma 9.3 is complete.

Set $\tilde{\Phi}=\hat{\Phi}-\Phi_{0}$. Then

$$
\begin{aligned}
& \triangle^{2} \tilde{\Phi}=0 \text { in } \mathbb{R}_{-}^{2} \backslash \bar{\Gamma}_{-1} \\
& \tilde{\Phi}=0, \frac{\partial^{2} \tilde{\Phi}}{\partial x^{2}}=0 \text { on }\{x=0\} \\
& \tilde{\Phi}=0, \frac{\partial \tilde{\Phi}}{\partial y}=0 \text { on } \Gamma_{-1}
\end{aligned}
$$


Take odd extension of $\tilde{\Phi}$ across $x=0$. This extension is biharmonic in

$$
\mathbb{R}_{0}^{2} \equiv \mathbb{R}_{-}^{2} \backslash\{(x, 0) ; x<-1 \text { or } x>1\}
$$

and

$$
\tilde{\Phi}=O\left(r^{1+\epsilon}\right) \text { if } r \rightarrow \infty, \quad \tilde{\Phi} \in H_{l o c}^{2}\left(\mathbb{R}^{2}\right)
$$

Let

$$
V_{0}(x, y)=\frac{1}{2} \int_{0}^{y} \triangle \tilde{\Phi}(x, s) d s .
$$

Since $\Delta \tilde{\Phi}=O\left(r^{-1 / 2}\right)$ near $( \pm 1,0)$, the integral is well defined in $\mathbb{R}_{0}^{2}$. It is clear that

$$
\frac{\partial}{\partial y} \Delta V_{0} \equiv 0
$$

and, consequently,

$$
\triangle V_{0} \equiv b_{0}(x) \text { in } \mathbb{R}_{0}^{2}
$$

Setting

$$
V_{1}(x)=\int_{0}^{x} \int_{0}^{\xi} b_{0}(s) d s d \xi
$$

the function $V \equiv V_{0}-V_{1}$ is harmonic in $\mathbb{R}_{0}^{2}$. One can easily verify that the function

$$
W=\tilde{\Phi}-y V
$$

is also harmonic in $\mathbb{R}_{0}^{2}$, and

$$
\tilde{\Phi}=y V+W \text { in } \mathbb{R}_{0}^{2}
$$

the function $V$ is bounded by $O\left(r^{\epsilon}\right)$ as $r \rightarrow \infty$, and $V(0, y)=0$. By the Phragmén-Lindelöf theorem we deduce that $V \rightarrow 0$ at $\infty$. It follows (by the maximum principle) that $V \equiv 0$. Since $W$ is harmonic and $W=W_{y}=0$ on $\{(x, 0) ; x<-1\}$ (since the same is true for $\tilde{\Phi}$ ), it follows that $W \equiv 0$. Hence $\hat{\Phi}-\Phi_{0} \equiv \tilde{\Phi} \equiv 0$.

We shall now prove the same result for $\beta_{\lambda, b}$, where $\beta_{\lambda, b}$ is defined by the expansion near $(\lambda, 0)$

$$
\psi_{\lambda, b}=2 \beta_{\lambda, b} r^{3 / 2}\left(\sin \frac{3}{2} \theta+\sin \frac{1}{2} \theta\right)+O\left(r^{5 / 2}\right),
$$

and $\psi_{\lambda, b}$ is the bounded solution in $H_{l o c}^{2}\left(D_{b}\right)$ to

$$
\begin{aligned}
& \triangle^{2} \psi=0 \text { in } D_{b} \backslash \bar{\Gamma}_{\lambda}, \\
& \psi=1, \quad \psi_{y}=0 \text { on } \partial D_{b}^{+} \backslash\{x=b\}, \\
& \psi=-1, \quad \psi_{y}=0 \text { on } \partial D_{b}^{-} \backslash\{x=b\}, \\
& \psi=-\frac{1}{2 a^{3}} y^{3}+\frac{3}{2 a} y, \psi_{x x}=0 \text { on }\{x=b\} \\
& \psi=0, \quad \psi_{y}=0 \text { on } \Gamma_{\lambda} .
\end{aligned}
$$

Notice that $\psi_{\lambda, b}$ is odd in $y$. As in (9.3),

$\beta_{\lambda, b}$ is continuous with respect to $(\lambda, b)$. 
Lemma 9.4. $\beta_{\lambda, b} \neq 0$ for $-\infty<\lambda<b<\infty$.

Proof. Let $\tilde{\psi}$ be the bounded solution in $H_{l o c}^{2}\left(D_{b}\right)$ satisfying (9.20) except for the boundary conditions on $\{x=b\}$ which are replaced by

$$
\tilde{\psi}_{x}=0, \quad \tilde{\psi}_{x x x}=0 .
$$

$\operatorname{Near}(\lambda, 0)$,

$$
\tilde{\psi}=2 \tilde{\beta} r^{3 / 2}\left(\sin \frac{3}{2} \theta+\sin \frac{1}{2} \theta\right)+O\left(r^{5 / 2}\right)
$$

As before,

$$
J \equiv \lim _{\epsilon \rightarrow} \int_{\partial B_{\epsilon}(\lambda, 0)}\left\{(\Delta \psi \cdot \Delta \tilde{\psi}) n_{1}-(\vec{s} \cdot \overrightarrow{\tilde{\Phi}}+\overrightarrow{\tilde{s}} \cdot \vec{\Phi})\right\}=16 \pi \beta_{\lambda, b} \tilde{\beta}
$$

and (by the path independence of $J$-integrals)

$$
J=\int_{\Lambda_{b, c}}\left\{(\Delta \psi \cdot \triangle \tilde{\psi}) n_{1}-(\vec{s} \cdot \overrightarrow{\tilde{\Phi}}+\overrightarrow{\tilde{s}} \cdot \vec{\Phi})\right\}
$$

If we perform integration by parts in the last integral and let $-c \rightarrow-\infty$, we obtain

$$
\begin{aligned}
J & =2 \int_{0}^{a}\left(\frac{\partial^{2} \psi}{\partial y^{2}} \frac{\partial^{2} \tilde{\psi}}{\partial y^{2}}\right)(-\infty, y) d y+\int_{-a}^{a}(\Delta \psi \cdot \Delta \tilde{\psi})(b, y) d y \\
& -\int_{-a}^{a}\left(\frac{\partial^{2} \psi}{\partial y^{2}} \triangle \tilde{\psi}+\frac{\partial^{2} \tilde{\psi}}{\partial y^{2}} \Delta \psi\right)(b, y) d y \\
& -\int_{-a}^{a}\left(\frac{\partial \psi}{\partial x} \frac{\partial}{\partial x} \triangle \tilde{\psi}+\frac{\partial \tilde{\psi}}{\partial x} \frac{\partial}{\partial x} \Delta \psi\right)(b, y) d y
\end{aligned}
$$

Using the boundary conditions at $x=b$, we find that

$$
J=\frac{18}{a^{3}}
$$

and upon comparing with (9.22) we conclude that $\beta_{\lambda, b} \neq 0$.

The proof of Lemma 9.2 shows that

$$
\left\|\psi_{\lambda, b}\right\|_{L^{\infty}} \leq C
$$

where $C$ is independent of $\lambda, b$. We shall need, however, also a gradient estimate:

Lemma 9.5. There holds:

$$
\left\|\nabla \psi_{\lambda, b}\right\|_{L^{\infty}\left(D_{b}\right)} \leq C
$$

where $C$ is a constant independent of $\lambda, b$.

Proof. Let

$$
\tilde{\psi}(x, y)=\psi_{\lambda, b}(x, y)-\left(-\frac{1}{2 a^{3}} y^{3}+\frac{3}{2 a} y\right)
$$


and extend $\tilde{\psi}$ into $\{x>b\}$ by odd reflection. Then

$$
\begin{aligned}
& \triangle^{2} \tilde{\psi}=0 \text { in } D \backslash\left\{\bar{\Gamma}_{\lambda} \cup \bar{\Gamma}_{-\lambda}^{*}\right\}, \\
& \tilde{\psi}=0, \quad \tilde{\psi}_{y}=0 \text { on } \partial D, \\
& \tilde{\psi}=0, \quad \tilde{\psi}_{y}= \begin{cases}-3 / 2 a & \text { on } \Gamma_{\lambda} \\
3 / 2 a & \text { on } \Gamma_{-\lambda}^{*}\end{cases}
\end{aligned}
$$

where $\Gamma_{-\lambda}^{*}$ is the reflection of $\Gamma_{\lambda}$ about $x=b$.

As easily verified (see [3]) the function

$$
q=|\nabla \tilde{\psi}|^{2}-(\triangle \tilde{\psi}) \tilde{\psi}
$$

is subharmonic in $D \backslash\left(\bar{\Gamma}_{\lambda} \cup \bar{\Gamma}_{-\lambda}^{*}\right)$ and, therefore,

$$
q(x, y) \leq \sup _{\partial\left\{D \backslash\left(\bar{\Gamma}_{\lambda} \cup \bar{\Gamma}_{-\lambda}^{*}\right)\right\}} q+\sup _{|y| \leq a} q( \pm \infty, y) .
$$

By the boundary conditions in (9.25) we see that the right-hand side of (9.26) is bounded by a constant $C$ independent of $\lambda, b$. Since $\tilde{\psi}(x, 0)=0(\tilde{\psi}$ is odd in $y),(9.26)$ implies that

$$
|\nabla \tilde{\psi}(x, 0)| \leq C, \quad \lambda<x<2 b-\lambda .
$$

We write $\tilde{\psi}$ in $\{0<y<a\}$ in the form $y W+\tilde{\psi}_{1}$ where $W$ is a bounded harmonic function satisfying the boundary conditions:

$$
W(x, 0)=\tilde{\psi}_{y}(x, 0), W(x, a)=0 ;
$$

then $\tilde{\psi}_{1}$ is a bounded biharmonic function satisfying:

$$
\begin{aligned}
& \tilde{\psi}_{1}=\frac{\partial}{\partial y} \tilde{\psi}_{1}=0 \text { at } y=0 \\
& \tilde{\psi}_{1} \text { and } \frac{\partial}{\partial y} \tilde{\psi}_{1} \text { are smooth at } y=a .
\end{aligned}
$$

Using (9.27) to estimate $\nabla(y W)$ and using elliptic estimates to estimate $\nabla \tilde{\psi}_{1}$, we find that

$$
\|\nabla \tilde{\psi}\|_{L^{\infty}(D \cap\{y>0\})} \leq C,
$$

from which the assertion (9.23) readily follows.

Lemma 9.6. For $\lambda$ close to $b, \beta_{\lambda, b}>0$.

Proof. Let $\delta=b-\lambda$ and choose a coordinate system with $(b, 0)$ at the origin. Consider the blow-up sequence

$$
\Psi_{\delta}(x, y)=\frac{1}{\delta} \tilde{\psi}(\delta x, \delta y) .
$$

Using Lemma 9.5 and elliptic estimates we deduce that

$$
\Psi_{\delta} \rightarrow \Psi_{0} \text { in } C_{l o c}^{0}\left(\overline{\mathbb{R}_{-}^{2}}\right)
$$


where $\Psi_{0}$ is a solution to

$$
\begin{aligned}
& \triangle^{2} \Psi_{0}=0 \text { in } \mathbb{R}_{-}^{2} \backslash \bar{\Gamma}_{-1} \\
& \Psi_{0}=0, \frac{\partial^{2} \Psi_{0}}{\partial x^{2}}=0 \text { on } x=0 \\
& \Psi_{0}=0, \frac{\partial \Psi_{0}}{\partial y}=-\frac{3}{2 a} \text { on } \Gamma_{-1} \text { (from both sides), }
\end{aligned}
$$

and

$$
\sup _{\mathbb{R}_{-}^{2}}\left|\nabla \Psi_{0}\right| \leq C<\infty
$$

Since $\Psi_{0}=O(r)$ as $r \rightarrow \infty$, it follows, from the proof that $\hat{\Phi}_{0} \equiv \Phi_{0}$, that such a solution is unique.

Let $w$ is the unique bounded solution to

$$
\begin{aligned}
& \triangle w=0 \text { in } \mathbb{R}_{-}^{2} \backslash \bar{\Gamma}_{-1} \\
& w=0 \text { on } x=0 \\
& w=-\frac{3}{2 a} \text { on } \Gamma_{-1} \text { (from both sides,); }
\end{aligned}
$$

then, by uniqueness,

$$
\Psi_{0}=y w .
$$

By the maximum principle $w>-3 / 2 a$, and therefore

$$
\hat{\Psi}_{0}(x, y) \equiv y w(x, y)+\frac{3}{2 a} y>0 \text { in }\{y>0\} .
$$

Since $\hat{\Psi}_{0}$ is biharmonic in $\mathbb{R}_{-}^{2} \backslash \bar{\Gamma}_{-1}$, odd in $y$, and

$$
\hat{\Psi}_{0}=\frac{\partial \hat{\Psi}_{0}}{\partial y}=0 \text { on } \Gamma_{-1}
$$

we have

$$
\hat{\Psi}_{0}=2 \beta_{0} r^{3 / 2}\left(\sin \frac{3}{2} \theta+\sin \frac{1}{2} \theta\right)+O\left(r^{5 / 2}\right)
$$

near $(-1,0)$ and, by $(9.32)$,

$$
\beta_{0}>0 \text {. }
$$

Introducing the function

$$
\hat{\Psi}_{\delta}=\Psi_{\delta}+\frac{3}{2 a} y
$$

and its expansion near $(-1,0)$ :

$$
\hat{\Psi}_{\delta}=2 \beta_{\delta} r^{3 / 2}\left(\sin \frac{3}{2} \theta+\sin \frac{1}{2} \theta\right)+O\left(r^{5 / 2}\right)
$$

we then have

$$
\beta_{\delta} \rightarrow \beta_{0}>0 \text { if } \delta \rightarrow 0 .
$$


On the other hand, from $(9.19),(9.24),(9.28),(9.29)$ and $(9.31),(9.32)$ we deduce that

$$
\beta_{\delta}=\beta_{\lambda, b} \delta^{1 / 2},
$$

and therefore $\beta_{\lambda, b}>0$ if $\lambda$ is close to $b$.

We have proved so far that

$\alpha_{\lambda, b}$ and $\beta_{\lambda, b}$ are continuous in $(\lambda, b)$ for $-\infty<\lambda<b<\infty$, and never vanish, and $\alpha_{\lambda, b}>0, \beta_{\lambda, b}>0$ if $b-\lambda$ is small. It follows that

$$
\alpha_{\lambda, b}>0 \quad \beta_{\lambda, b}>0 \text { for all }-\infty<\lambda<b<\infty \text {. }
$$

Taking $\lambda=0$ and $b \rightarrow \infty$ we deduce that

$$
\alpha=\lim \alpha_{0, b} \geq 0, \beta=\lim \beta_{0, b} \geq 0 .
$$

Recalling (8.17), (8.19) we see that $\alpha>0, \beta>0$ and thus, by (8.15), $\dot{y}(0+)>0$.

\section{References}

[1] R.A. Adams, Sobolev Spaces, Academic Press, New York (1975).

[2] S. Agmon, A. Douglis and L. Nirenberg, Estimates near the boundary for solutions of elliptic partial differential equations satisfying general boundary conditions, I, Comm. Pure Appl. Math., 12 (1959), 623-727.

[3] R.J. Duffin, The maximum principle and biharmonic functions, J. Math. Anal. Appl., 3 (1961), 399-405.

[4] L.B. Freund, Dynamic Fracture Mechanics, Cambridge University Press, Cambridge, 1990.

[5] A. Friedman and W. Liu, A system of partial differential equations arising in electrophotography, J. Diff. Eqs., 89 (1991), 272-304.

[6] R.D. Gregory, The semi-infinite strip $x \leq 0,-1 \leq y \leq 1$; completeness of the Papkovich-Fadle eigenfunctions when $\phi_{x x}(0, y), \phi_{y y}(0, y)$ are prescribed, J. Elasticity, 10 (1980), 57-80.

[7] A.A. Griffith, The phenomenon of rupture and flow in solid, Philos. Trans. Royal. Soc. London, A221 (1920), 163-198.

[8] M.E. Gurtin, On the energy release rate in quasi-static elastic crack propagation, J. Elasticity, 9 (1979), 187-195.

[9] P.D. Lax, A Phragmén-Lindelöf theorem in harmonic analysis and its application to some questions in the theory of elliptic equations, Comm. Pure Appl. Math., 10 (1957), 361-189.

[10] K. Ohtsuka, Generalized $J$-integral and three dimensional fracture mechanics, I, II, Hiroshima Math. J., 11 (1981), 21-52; 16 (1986), 327-352.

[11] J.L. Rice, Mathematical analysis in mechanics of fracture, in Fracture, Vol. 2 , ed. H. Liebowitz, Academic Press, New York, 11 (1968), 191-311.

[12] L.I. Slepyan, Principle of maximum energy dissipation rate in crack dynamics, J. Mech. Phys. Solids, 41 (1993), 1019-1033.

[13] H. Stump and K.C. Le, Variational formulation of the crack problem for an elastoplastic body at finite strain, Z. Angew. Math. Mech., 72 (1992), 387-196.

[14] D. Vasilopoulos, On the determination of higher order terms of singular elastic stress fields near corners, Numer. Math., 53 (1988), 51-95. 

\#
Author/s
Title

Recent IMA Preprints

1202 Joel Spencer, The Erdös-Hanani conjecture via Talagrand's inequality

1203 Zhangxin Chen, Superconvergence results for Galerkin methods for wave propagation in various porous media

1204 Russell Lyons, Robin Pemantle \& Yuval Peres, When does a branching process grow like its mean? Conceptual proofs of $L \log L$ criteria

1205 Robin Pemantle, Maximum variation of total risk

1206 Robin Pemantle \& Yuval Peres, Galton-Watson trees with the same mean have the same polar sets

1207 Robin Pemantle, A shuffle that mixes sets of any fixed size much faster than it mixes the whole deck

1208 Itai Benjamini, Robin Pemantle \& Yuval Peres, Martin capacity for Markov chains and random walks in varying dimensions

1209 Wlodzimierz Bryc \& Amir Dembo, On large deviations of empirical measures for stationary Gaussian processes

1210 Martin Hildebrand, Some random processes related to affine random walks

1211 Alexander E. Mazel \& Yurii M. Suhov, Ground states of a Boson quantum lattice model

1212 Roger L. Fosdick \& Darren E. Mason, Single phase energy minimizers for materials with nonlocal spatial dependence

1213 Bruce Hajek, Load balancing in infinite networks

1214 Petr Klouček, The transonic flow problems stability analysis and numerical results

1215 Petr Klouček, On the existence of the entropic solutions for the transonic flow problem

1216 David A. Schmidt \& Chjan C. Lim, Full sign-invertibility and symplectic matrices

1217 Piermarco Cannarsa \& Maria Elisabetta Tessitore, Infinite dimensional Hamilton-Jacobi equations and Dirichlet boundary control problems of parabolic type

1218 Zhangxin Chen, Multigrid algorithms for mixed methods for second order elliptic problems

1219 Zhangxin Chen, Expanded mixed finite element methods for linear second order elliptic problems I

1220 Gang Bao, A note on the uniqueness for an inverse diffraction problem

1221 Moshe Fridman, A two state capital asset pricing model

1222 Paolo Baldi, Exact asymptotics for the probability of exit from a domain and applications to simulation

1223 Carl Dou \& Martin Hildebrand, Enumeration and random random walks on finite groups

1224 Jaksa Cvitanic \& Ioannis Karatzas, On portfolio optimization under "drawdown" constraints

1225 Avner Friedman \& Yong Liu, A free boundary problem arising in magnetohydrodynamic system

1226 Dominic Welsh, Randomised approximation schemes for Tutte-Gröthendieck invariants

1227 Zhangxin Chen, Bernardo Cockburn. Carl L. Gardner, \& Joseph W. Jerome, Quantum hydrodynamic simulation of hysteresis in the resonant tunneling diode

1228 E.G. Kalnins, G.C. Williams, \& Willard Miller, Jr., Intrinsic characterisation of the separation constant for spin one and gravitational perturbations in Kerr geometry

1229 Zhangxin Chen, Large-scale averaging analysis of multiphase flow in fractured reservoirs

1230 Bruce Hajek \& Babu Narayanan, Multigraphs with the most edge covers

1231 K.B. Athreya, Entropy maximization

1232 F.I. Karpelevich \& Yu.M. Suhov, Functional equations in the problem of boundedness of stochastic branching dynamics

1233 E. Dibenedetto \& V. Vespri, On the singular equation $\beta(u)_{t}=\Delta u$

1234 M.Ya. Kelbert \& Yu.M. Suhov, The Markov branching random walk and systems of reaction-diffusion (KolmogorovPetrovskii-Piskunov) equations

1235 M. Hildebrand, Random walks on random regular simple graphs

1236 W.S. Don \& A. Solomonoff, Accuracy enhancement for higher derivatives using Chebyshev collocation and a mapping technique

1237 D. Gurarie, Symmetries and conservation laws of two-dimensional hydrodynamics

1238 Z. Chen, Finite element methods for the black oil model in petroleum reservoirs

1239 G. Bao \& A. Friedman, Inverse problems for scattering by periodic structure

1240 G. Bao, Some inverse problems in partial differential equations

1241 G. Bao, Diffractive optics in periodic structures: The TM polarization

1242 C.C. Lim \& D.A. Schmidt, On noneven digraphs and symplectic pairs

1243 H.M. Soner, S.E. Shreve \& J. Cvitanić. There is no nontrivial hedging portfolio for option pricing with transaction costs

1244 D.L. Russell \& B-Yu Zhang, Exact controllability and stabilizability of the Korteweg-de Vries equation

1245 B. Morton, D. Enns \& B-Yu Zhang. Stability of dynamic inversion control laws applied to nonlinear aircraft pitch-axis models

1246 S. Hansen \& G. Weiss, New results on the operator Carleson measure criterion

1247 V.A. Malyshev \& F.M. Spieksma, Intrinsic convergence rate of countable Markov chains

1248 G. Bao, D.C. Dobson \& J.A. Cox, Mathematical studies in rigorous grating theory 
1249 G. Bao \& W.W. Symes, On the sensitivity of solutions of hyperbolic equations to the coefficients

1250 D.A. Huntley \& S.H. Davis, Oscillatory and cellular mode coupling in rapid directional solidification

1251 M.J. Donahue, L. Gurvits, C. Darken \& E. Sontag, Rates of convex approximation in non-Hilbert spaces

1252 A. Friedman \& B. Hu, A Stefan problem for multi-dimensional reaction diffusion systems

1253 J.L. Bona \& B-Y. Zhang, The initial-value problem for the forced Korteweg-de Vries equation

1254 A. Friedman \& R. Gulliver, Organizers, Mathematical modeling for instructors

1255 S. Kichenassamy, The prolongation formula for tensor fields

1256 S. Kichenassamy, Fuchsian equations in Sobolev spaces and blow-up

1257 H.S. Dumas, L. Dumas, \& F. Golse, On the mean free path for a periodic array of spherical obstacles

1258 C. Liu, Global estimates for solutions of partial differential equations

1259 C. Liu, Exponentially growing solutions for inverse problems in PDE

1260 Mary Ann Horn \& I. Lasiecka, Nonlinear boundary stabilization of parallelly connected Kirchhoff plates

1261 B. Cockburn \& H. Gau, A posteriori error estimates for general numerical methods for scalar conservation laws

1262 B. Cockburn \& P-A. Gremaud, A priori error estimates for numerical methods for scalar conservation laws. Part I: The general approach

1263 R. Spigler \& M. Vianello, Convergence analysis of the semi-implicit euler method for abstract evolution equations

1264 R. Spigler \& M. Vianello, WKB-type approximation for second-order differential equations in $C^{*}$-algebras

1265 M. Menshikov \& R.J. Williams, Passage-time moments for continuous non-negative stochastic processes and applications

1266 C. Mazza, On the storage capacity of nonlinear neural networks

1267 Z. Chen, R.E. Ewing \& R. Lazarov, Domain decomposition algorithms for mixed methods for second order elliptic problems

1268 Z. Chen, M. Espedal \& R.E. Ewing, Finite element analysis of multiphase flow in groundwater hydrology

1269 Z. Chen, R.E. Ewing, Y.A. Kuznetsov, R.D. Lazarov \& S. Maliassov, Multilevel preconditioners for mixed methods for second order elliptic problems

1270 S. Kichenassamy \& G.K. Srinivasan, The structure of WTC expansions and applications

1271 A. Zinger, Positiveness of Wigner quasi-probability density and characterization of Gaussian distribution

1272 V. Malkin \& G. Papanicolaou, On self-focusing of short laser pulses

1273 J.N. Kutz \& W.L. Kath, Stability of pulses in nonlinear optical fibers using phase-sensitive amplifiers

1274 S.K. Patch, Recursive recovery of a family of Markov transition probabilities from boundary value data

1275 C. Liu, The completeness of plane waves

1276 Z. Chen \& R.E. Ewing, Stability and convergence of a finite element method for reactive transport in ground water

1277 Z. Chen \& Do Y. Kwak, The analysis of multigrid algorithms for nonconforming and mixed methods for second order elliptic problems

1278 Z. Chen, Expanded mixed finite element methods for quasilinear second order elliptic problems II

1279 M.A. Horn \& W. Littman, Boundary control of a Schrödinger equation with nonconstant principal part

1280 R.E. Ewing, Yu.A. Kuznetsov, R.D. Lazarov \& S. Maliassov, Substructuring preconditioning for finite element approximations of second order elliptic problems. I. Nonconforming linear elements for the Poisson equation in a parallelepiped

1281 S. Maliassov, Substructuring preconditioning for finite element approximations of second order elliptic problems. II. Mixed method for an elliptic operator with scalar tensor

1282 V. Jakšić \& C.-A. Pillet, On model for quantum friction II. Fermi's golden rule and dynamics at positive temperatures 1283 V. M. Malkin, Kolmogorov and nonstationary spectra of optical turbulence

1284 E.G. Kalnins, V.B. Kuznetsov \& W. Miller, Jr., Separation of variables and the XXZ Gaudin magnet

1285 E.G. Kalnins \& W. Miller, Jr., A note on tensor products of $q$-algebra representations and orthogonal polynomials

1286 E.G. Kalnins \& W. Miller, Jr., $q$-algebra representations of the Euclidean, pseudo-Euclidean and oscillator algebras, and their tensor products

1287 L.A. Pastur, Spectral and probabalistic aspects of matrix models

1288 K. Kastella, Discrimination gain to optimize detection and classification

1289 L.A. Peletier \& W.C. Troy, Spatial patterns described by the Extended Fisher-Kolmogorov (EFK) equation: Periodic solutions

1290 A. Friedman \& Y. Liu, Propagation of cracks in elastic media

1291 A. Friedman \& C. Huang, Averaged motion of charged particles in a curved strip

1292 G. R. Sell, Global attractors for the 3D Navier-Stokes equations

1293 C. Liu, A uniqueness result for a general class of inverse problems

1294 H-O. Kreiss, Numerical solution of problems with different time scales II

1295 B. Cockburn, G. Gripenberg, S-O. Londen, On convergence to entropy solutions of a single conservation law

1296 S-H. Yu, On stability of discrete shock profiles for conservative finite difference scheme

1297 H. Behncke \& P. Rejto, A limiting absorption principle for separated Dirac operators with Wigner Von Neumann type potentials 